\title{
Precise asymptotics of the Ricci flow neckpinch
}

\author{
Sigurd B. Angenent and Dan Knopf
}

The best known finite-time local Ricci flow singularity is the neckpinch, in which a proper subset of the manifold becomes geometrically close to a portion of a shrinking cylinder. In this paper, we prove precise asymptotics for rotationally-symmetric Ricci flow neckpinches. We then compare these rigorous results with formal matched asymptotics for fully general neckpinch singularities.

1 Introduction

1.1 Antecedents

1.2 Overview

\section{$1.3 \quad$ A diameter bound}

2 Rigorous asymptotics of the $\mathrm{SO}\left(\mathbb{R}^{n+1}\right)$ invariant neckpinch

2.1 The blown-up radius and the linearized equation

2.2 Pointwise estimates for $u$ and $v$

2.3 Transition to commuting variables

S.B.A. was partially supported by NSF grant DMS-0101124. D.K. was partially supported by NSF grants DMS-0511184, DMS-0505920, and a University of Texas Summer Research Assignment. 
$\begin{array}{lll}2.7 & \text { Decay of } a_{0} & 788\end{array}$

$\begin{array}{lll}2.8 & \text { Decay of } W & 789\end{array}$

$\begin{array}{lll}2.9 & \text { Derivative estimates for } W & \mathbf{7 9 0}\end{array}$

$\begin{array}{ll}2.10 \text { A bound for }\|\sigma W\| & 792\end{array}$

$\begin{array}{lll}2.11 & \text { Pointwise estimates for } W & 793\end{array}$

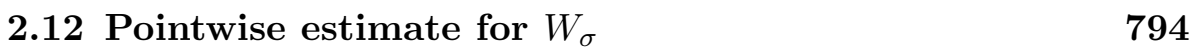

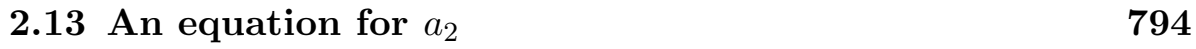

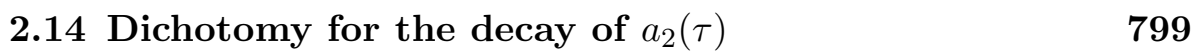

$\begin{array}{lr}2.15 \text { Pinching time } & 800\end{array}$

\begin{tabular}{ll}
2.16 & Initial data \\
\hline
\end{tabular}

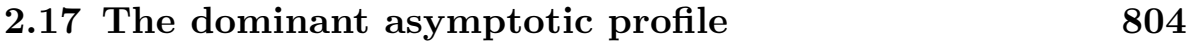

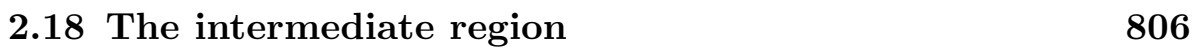

$\begin{array}{lr}2.19 \text { Convergence in the outer region } & 812\end{array}$

$\begin{array}{lll}2.20 & \text { Asymptotics in the outer region } & 816\end{array}$

$\begin{array}{ll}3 & \text { Formal matched asymptotics for general } \\ \text { neckpinches } & 817\end{array}$

$\begin{array}{llr}3.1 & \text { The cylinder soliton } & 819\end{array}$

$\begin{array}{lll}3.2 & \text { Dilated Ricci flow } & 821\end{array}$

$\begin{array}{lll}\text { 3.3 } & \text { First and second order variation formulas } & 822\end{array}$

3.4 Arbitrary perturbations of a stationary solution $\quad 824$

$\begin{array}{lll}3.5 & \text { The linearization at the cylinder soliton } & 825\end{array}$ 


\subsection{An upper bound for the spectrum of the linearization}

3.8 The spectrum of the linearization

3.10 The inner layer $|x|=\mathrm{o}(\sqrt{\tau})$

3.11 The intermediate layer $|x|=\mathcal{O}(\sqrt{\tau})$

3.12 The outer layer $|x|=o(1 / \sqrt{T-t})$

\section{Introduction}

\subsection{Antecedents}

In virtually all known applications of Ricci flow, it is valuable to have a good understanding of singularity formation. Heuristically, there are at least three reasons for this. The first is that one expects finite-time singularities to form for a broad spectrum of initial data. Indeed, such singularities are inevitable if the scalar curvature is ever strictly positive. The second reason is that one expects the geometry of a solution to resemble a standard model (for example, a self-similar solution) in a space-time neighborhood of a developing singularity. The third reason is that having a sufficiently detailed picture of a developing singularity facilitates the geometric topological surgeries by which Ricci flow decomposes a given manifold.

Whenever a compact solution $\left(M^{n}, g(\cdot)\right)$ of Ricci flow encounters a singularity at time $T<\infty$, standard short-time existence results imply that

$$
\lim _{t \nearrow T} \max _{x \in M^{n}}|\operatorname{Rm}(x, t)|=\infty
$$

In fact, a finite-time singularity happens if and only if

$$
\limsup _{t \nearrow T} \max _{x \in M^{n}}|\operatorname{Rc}(x, t)|=\infty \text {. }
$$


This claim follows by a short argument from a result of Simon [31]; ${ }^{1}$ recently, Šešum gave an independent, direct proof [29]. The most interesting cases are those where a local singularity forms, that is, where there exists an open set $\Omega \subset M^{n}$ such that

$$
\sup _{\Omega \times[0, T)}|\operatorname{Rc}(x, t)|<\infty .
$$

The first rigorous constructions of local singularities were done by Simon [30]. Here the manifold is a noncompact warped product $\mathbb{R} \times_{f} S^{n}$, and a supersolution of the Ricci flow PDE is used to prove that $f$ vanishes in finite time on a proper subset of $\mathbb{R}$. A second class of examples is constructed in [14]. Here the manifold is a complex line bundle $\mathbb{C} \hookrightarrow \mathbb{L}_{-k}^{n} \rightarrow \mathbb{C} \mathbb{P}^{n-1}$ with $1 \leq k \leq n-1$. As the singularity forms, the flow performs an algebraicgeometric blow-down of the $\mathbb{C} \mathbb{P}^{n-1}$, while the rest of the manifold converges locally smoothly to a metric cone on $\left(\mathbb{C}^{n} \backslash\{0\}\right) / \mathbb{Z}_{k}$.

As Perelman writes, "the most natural way of forming a singularity in finite time is by pinching an (almost) round cylindrical neck" [29]. This is the kind of local singularity that we analyze in this paper. It is a continuation of our earlier work [3], where we gave the first rigorous examples of local singularity formation on compact manifolds by constructing neckpinches for rotationally symmetric metrics on $S^{n+1}$. In that paper, we obtained local $a$ priori estimates for the space-time scales at which a developing neckpinch singularity resembles the self-similarly hrinking cylinder soliton. The present paper proves that those estimates are sharp and gives precise asymptotics for neckpinch formation.

When considering a local singularity, two natural and important questions arise. (1) What is the nature of the set of points in space on which the metric becomes singular? (2) What is the asymptotic behavior of the solution near this set as the singularity time is approached? In the past two decades, a rich literature of both rigorous and formal matched asymptotics has developed for analyzing the local behavior of singular solutions of

\footnotetext{
${ }^{1}$ Let $\left(\mathcal{M}^{n}, g(t)\right)$ be a solution of Ricci flow on a compact manifold. Assume $g(t)$ is smooth for $t \in[0, T)$, where $T<\infty$. If $\lim \sup _{t} \nearrow_{T}\left(\max _{x \in \mathcal{M}^{n}}|\operatorname{Rc}(x, t)|\right)<\infty$, then $\left[21\right.$, Lemma 14.2] guarantees existence of a complete $C^{0}$ limit metric $g(T)$. One may then apply [31, Theorem 1.1], choosing a background metric $\bar{g}:=g(T-\delta)$ such that $(1-\varepsilon) \bar{g} \leq g \leq(1+\varepsilon) \bar{g}$, where $\varepsilon=\varepsilon(n)$ and $\delta=\delta(\varepsilon)$. Let $\bar{K}=\max _{x \in \mathcal{M}^{n}}|\operatorname{Rm}(\bar{g})|_{\bar{g}}$. Simon's theorem implies that there exists a $\eta=\eta(n, \bar{K})$ such that for any $\theta \in$ $[0, \delta]$, a solution $\hat{g}(s)$ of harmonic-map-coupled Ricci flow exists for $0 \leq s<\eta$ and satisfies $\hat{g}(0)=g(T-\theta)$; moreover, $\hat{g}(s)$ is smooth for $0<s<\eta$. Since harmonicmap-coupled Ricci flow is equivalent to Ricci flow modulo diffeomorphisms, the claim follows by taking $\theta=\eta / 2$.
} 
nonlinear PDE such as $u_{t}=\Delta(\log u)$ or $u_{t}=\Delta u+F(u)$, where $F(u)=u^{p}$ or $F(u)=e^{u}$. Some of the many noteworthy results of this type are given in $[7,15-20,22,23,26,27,34]$. A few results specific to geometric evolution equations such as mean curvature flow and harmonic map flow are given in $[1,2,4,5,10,24,33]$.

\subsection{Overview}

This paper is divided into two main parts.

Rigorous asymptotics. In Section 2, we derive rigorous asymptotics for rotationally symmetric neckpinches. The set-up is as follows. Let $g_{\text {can }}$ denote the round metric of radius 1 on $S^{n}$. Then any $\mathrm{SO}(n+1)$-invariant metric on $S^{n+1}$ can be written as

$$
g=\varphi^{2} d x^{2}+\psi^{2} g_{\text {can }}
$$

on $(-1,1) \times S^{n}$, which may be naturally identified with the sphere $S^{n+1}$ with its north and south poles removed. The quantity $\psi(x, t)>0$ may thus be regarded as the radius of the hypersurface $\{x\} \times S^{n}$ at time $t$. It is natural to write geometric quantities related to $g$ in terms of the distance $s(x)=$ $\int_{0}^{x} \varphi(\hat{x}) d \hat{x}$ from the equator. ${ }^{2}$ Then one can write the metric in the nicer form of a warped product

$$
g=d s^{2}+\psi^{2} g_{\text {can }}
$$

bearing in mind that $s$ is ultimately a function of both $x$ and $t$. (Note that one encounters the commutator $\left[\partial_{t}, \partial_{s}\right]=-n \psi^{-1} \psi_{s s} \partial_{s}$ when taking derivatives with $x$ held constant.)

In [3], we established neckpinching for a class of rotationally symmetric data essentially described by three conditions: (1) the initial metric should have positive scalar curvature on all of $S^{n+1}$ and positive Ricci curvature on the "polar caps", in the terminology of [3]; (2) its sectional curvature should be positive on planes tangential to the spheres $\{x\} \times S^{n}$; and (3) it should be "sufficiently pinched", i.e., the minimum radius should be sufficiently small relative to the maximum radius. In this paper, we impose an additional hypothesis: (4) the initial metric should be reflection symmetric, i.e., $\psi(s, 0) \equiv \psi(-s, 0)$. Under these hypotheses, the results we obtain

\footnotetext{
${ }^{2}$ This also has the effect of fixing a gauge, thereby breaking the diffeomorphism invariance of Ricci flow and making the resulting equations strictly parabolic.
} 
in Section 2 (combined with results from the predecessor [3] to this paper) imply that the solution obeys a precise asymptotic profile.

To describe this profile requires some additional notation. If $T<\infty$ is the singularity time, let $u=\psi / \sqrt{2(n-1)(T-t)}$ be the blown-up radius defined below in $(2.2)$; let $\sigma=s / \sqrt{T-t}$ be the rescaled distance to the neck defined in (2.3); and let $\tau=-\log (T-t)$ denote the rescaled time variable defined in $(2.5)$. Note that $(\sigma, \tau)$ are self-similar coordinates with respect to the blown-up flow. We summarize our results as

Theorem 1.1. For an open set of initial metrics symmetric with respect to reflection and rotation, the solution $\left(S^{n+1}, g(t)\right)$ of Ricci flow becomes singular at $T<\infty$. Its diameter remains bounded for all $t \in[0, T)$. The singularity occurs only on the hypersurface $\{0\} \times S^{n}$. The solution satisfies the following asymptotic profile.

Inner region. On any interval $|\sigma| \leq A$, one has

$$
u(\sigma, \tau)=1+\frac{\sigma^{2}-2}{8 \tau}+\mathrm{o}\left(\frac{1}{\tau}\right) \quad \text { uniformly as } \tau \rightarrow \infty .
$$

Intermediate region. On any interval $A \leq|\sigma| \leq B \sqrt{\tau}$, one has

$$
u(\sigma, \tau)=\sqrt{1+(1+\mathrm{o}(1)) \frac{\sigma^{2}}{4 \tau}} \quad \text { uniformly as } \tau \rightarrow \infty .
$$

Outer region. For any $\varepsilon>0$, there exist $C<\infty$ and $\bar{t}<T$ such that

$$
\left(\frac{1}{2} \sqrt{n-1}-\varepsilon\right) \frac{s}{\sqrt{\log (1 / s)}} \leq \psi(s, t) \leq\left(\frac{1}{2} \sqrt{n-1}+\varepsilon\right) \frac{s}{\sqrt{\log (1 / s)}}
$$

for all points on the neck such that $|\sigma| \geq C \sqrt{\tau}$ and $t \in(\bar{t}, T)$.

Formal asymptotics. In Section 3, we derive formal matched asymptotics for fully general neckpinches, without any symmetry assumptions whatsoever. These are analogous to the formal asymptotics for $m=2$ (presumably typical) case of MCF singularities considered in [5]. Our method here is to study arbitrary perturbations of the self-similar cylinder soliton. That is, we consider $\tilde{g}=g+h$, where

$$
g=d x^{2}+2(n-1)(T-t) g_{\text {can }}
$$

is the cylinder soliton on $\mathbb{R} \times S^{n}$, and $h$ is an arbitrary (small) (2,0)-tensor. To accomplish our analysis, we modify the Ricci flow so that the soliton 
becomes a fixed point of an equivalent but strictly parabolic flow. Because the linearization of that flow has a null eigenvalue, one expects to find a center manifold. We proceed by carrying out a quadratic variational analysis in order formally to compute the dynamics of the flow on that center manifold. That is to say, we formally analyze all solutions that exhibit the $\tau^{-1}$ rate of convergence that is proved for rotationally symmetric solutions in Theorem 1.1.

The computations involved are extensive, but the conclusion we obtain is quite satisfying: the formal matched asymptotics suggest that the behavior analyzed rigorously in Section 2 should indeed be stable for fully general Ricci flow neckpinches. See the introduction to Section 3 for a more complete discussion.

\subsection{A diameter bound}

We conclude this introduction with an observation that follows directly from the estimates obtained in [3]. This result confirms an expectation of Perelman (R.S. Hamilton, private communication).

Lemma 1.2. Let $\left(S^{n+1}, g(t)\right)$ be any $\mathrm{SO}(n+1)$-invariant solution of Ricci flow such that $g(0)$ has positive scalar curvature and positive sectional curvature on planes tangential to the spheres $\{x\} \times S^{n}$.

Assume, in the language of [3], that the metric $g(t)$ has at least two bumps for all $t<T$. Let $x=a(t)$ and $x=b(t)$ be the locations of the leftand right-most bumps, and assume that for all $t<T$ one has $\psi(a(t), t) \geq c$, $\psi(b(t), t) \geq c$ for some constant $c>0$.

If $g(t)$ becomes singular at $T<\infty$, then $\operatorname{diam}\left(S^{n+1}, g(t)\right)$ remains bounded as $t \nearrow T$.

Proof. By [3, Proposition 5.4], the limit profile $\psi(\cdot, T)$ exists. Let $a(t)<b(t)$ in $(-1,1)$ denote the left-most and right-most bumps, respectively. Then $a(t) \rightarrow a(T)$ and $b(t) \rightarrow b(T)$. By [3, Lemma 5.6], the Ricci curvature is positive (so distances are decreasing) on $(-1, a(t)]$ and $[b(t), 1)$. Hence it will suffice to bound dist ${ }_{g(t)}\left(x_{1}, x_{2}\right)$ for arbitrary $x_{1}<x_{2}$ in the interval $(a(T)-\varepsilon, b(T)+\varepsilon) \subset(-1,1)$. 
Equations (5) and (11) of [3] imply that

$$
\begin{aligned}
\frac{d}{d t} \operatorname{dist}_{g(t)}\left(x_{1}, x_{2}\right) & =\frac{d}{d t} \int_{x_{1}}^{x_{2}} \varphi(x, t) d x \\
& =n \int_{s\left(x_{1}\right)}^{s\left(x_{2}\right)} \frac{\psi_{s s}}{\psi} d s \\
& =n\left\{\left.\frac{\psi_{s}}{\psi}\right|_{s\left(x_{1}\right)} ^{s\left(x_{2}\right)}+\int_{s\left(x_{1}\right)}^{s\left(x_{2}\right)}\left(\frac{\psi_{s}}{\psi}\right)^{2} d s\right\} .
\end{aligned}
$$

Proposition 5.1 of [3] bounds $\psi_{s}$ uniformly, while [3, Lemma 5.5] shows that the number of bumps and necks is nonincreasing in time. It follows that

$$
\int_{s\left(x_{1}\right)}^{s\left(x_{2}\right)}\left(\frac{\psi_{s}}{\psi}\right)^{2} d s \leq C \int_{s\left(x_{1}\right)}^{s\left(x_{2}\right)} \frac{\left|\psi_{s}\right|}{\psi^{2}} d s \leq C\left[\frac{1}{\psi_{\min }(t)}-\frac{1}{\psi_{\max }(t)}\right] \leq \frac{C}{\psi_{\min }(t)} .
$$

Hence [3, Lemma 6.1] allows us to conclude that

$$
\left|\frac{d}{d t} \operatorname{dist}_{g(t)}\left(x_{1}, x_{2}\right)\right| \leq \frac{C}{\psi_{\min }(t)} \leq \frac{C}{\sqrt{T-t}},
$$

which is integrable.

Recalling, [3, Lemmata 7.1 and 10.1], one immediately obtains

Corollary 1.3. Assume that $\left(S^{n+1}, g(t)\right)$ satisfies the hypotheses of Lemma 1.2 and is reflection-symmetric. Then the final-time profile $\psi(\cdot, T)$ has $\psi(x, T)$ strictly positive for all $x \neq 0$. Because $|\mathrm{Rm}| \leq C / \psi^{2}$, this implies that the singularity occurs only on the hypersurface $\{0\} \times S^{n}$.

Diameter estimates for arbitrary finite-time Ricci flow singularities are studied from another point of view in [32]. Lower bounds for diameter are known for certain topologies; see [25].

\section{Rigorous asymptotics of the $\mathrm{SO}\left(\mathbb{R}^{n+1}\right)$ invariant neckpinch}

\subsection{The blown-up radius and the linearized equation}

The radius $\psi$ satisfies

$$
\psi_{t}=\psi_{s s}-(n-1) \frac{1-\psi_{s}^{2}}{\psi} .
$$


We will assume that the solution is defined on some time interval $t_{0} \leq t<T$, where the initial time $t_{0}$ need not be 0 . In order to construct examples of solutions that follow our precise asymptotics, we will at some point actually have to assume that $T-t_{0}$ is "sufficiently small".

We consider the blown-up radius

$$
u \doteqdot \frac{\psi}{R_{n}(t)}, \quad \text { where } R_{n}(t) \doteqdot \sqrt{2(n-1)(T-t)}
$$

in which $T$ may or may not be the blow-up time. We also introduce the rescaled distance to the neck

$$
\sigma \doteqdot \frac{s}{\sqrt{T-t}}
$$

(Here the absence of a factor $n-1$ in $\sqrt{T-t}$ is intentional and will result in the numerically simplest equations later on.) Then we have

$$
\psi_{s}=\sqrt{2(n-1)} u_{\sigma}, \quad \psi_{s s}=\sqrt{2(n-1)} \frac{u_{\sigma \sigma}}{\sqrt{T-t}}
$$

and

$$
\psi_{t}=\sqrt{2(n-1)(T-t)} u_{t}-\frac{\sqrt{2(n-1)}}{2 \sqrt{T-t}} u
$$

This leads to the following evolution equation for $u$,

$$
u_{\tau}=u_{\sigma \sigma}+\frac{1}{2}\left(u-\frac{1}{u}\right)+(n-1) \frac{u_{\sigma}^{2}}{u},
$$

where

$$
\tau \doteqdot-\log (T-t)
$$

The blown-up time variable $\tau$ takes values in the interval

$$
\tau_{0} \leq \tau<\infty, \quad \text { where } \tau_{0} \doteqdot-\log \left(T-t_{0}\right)
$$

Without loss of generality, we will assume that $\tau_{0} \geq 1$. 
Near the neck, $u$ will be close to the self-similar radius $u \approx 1$. So to linearize, we set

$$
u=1+v
$$

and observe that $v$ must satisfy

$$
v_{\tau}=v_{\sigma \sigma}+v+\frac{(n-1) v_{\sigma}^{2}-1 / 2 v^{2}}{1+v} .
$$

Here the partial derivatives $\partial / \partial \sigma$ and $\partial / \partial \tau$ do not commute. One has instead

$$
\left[\partial_{t}, \partial_{s}\right]=-n \frac{\psi_{s s}}{\psi} \partial_{s}
$$

and thus

$$
\left[\partial_{\tau}, \partial_{\sigma}\right]=\left[(T-t) \partial_{t}, \sqrt{T-t} \partial_{s}\right]=-\left\{\frac{1}{2}+n \frac{u_{\sigma \sigma}}{u}\right\} \partial_{\sigma}
$$

\subsection{Pointwise estimates for $u$ and $v$}

In [3], we showed that on solutions of Ricci flow whose scalar curvature is non-negative, the quantity

$$
F=\frac{K}{L}\left\{2+\log \frac{L}{L_{\min }(0)}\right\}
$$

satisfies a maximum principle. Here

$$
K=\frac{\psi_{s s}}{\psi} \quad \text { and } \quad L=\frac{1-\psi_{s}^{2}}{\psi^{2}}
$$

and

$$
L_{\min }(0)=\left.\inf \frac{1-\psi_{s}^{2}}{\psi^{2}}\right|_{t=0} .
$$

The maximum principle implies that $\sup F$ does not increase when it is above $n-1$.

Let us assume throughout this paper that the initial metric has nonnegative scalar curvature, and that

$$
\sup F \leq F^{*}
$$

for some fixed constant $F^{*} \geqslant n-1$. 
When one writes the quantity $F$ in terms of $\psi, \psi_{s}$ and $\psi_{s s}$, the estimate $F \leq F^{*}$ implies the following upper bounds for $\psi, \psi_{s}$ and $\psi_{s s}$ in terms of the radius of the neck $\psi(0, t)$.

Lemma 2.1. There exist constants $\delta>0$ and $C<\infty$ that only depend on $F^{*}$ and the dimension $n$ such that

$$
1-\frac{C}{\tau} \leq u \leq 1+C \frac{1+\sigma^{2}}{\tau} \quad \text { for }|\sigma| \leq 2 \sqrt{ } \tau
$$

and

$$
1-\frac{C}{\tau} \leq u \leq C \frac{|\sigma|}{\sqrt{ } \tau} \sqrt{\log \frac{|\sigma|}{\sqrt{ } \tau}} \quad \text { for } 2 \sqrt{ } \tau \leq|\sigma| \leq e^{\delta \tau}
$$

For the derivative $u_{\sigma}$, we have

$$
\begin{gathered}
\left|u_{\sigma}\right| \leq C \frac{1+|\sigma|}{\tau} \quad \text { for }|\sigma| \leq 2 \sqrt{ } \tau \\
\left|u_{\sigma}\right| \leq \frac{C}{\sqrt{ } \tau} \sqrt{\log \frac{|\sigma|}{\sqrt{ } \tau}} \quad \text { for } 2 \sqrt{ } \tau \leq|\sigma| \leq e^{\delta \tau}
\end{gathered}
$$

In both cases, these estimates imply that $\left|u_{\sigma}\right| \leq C \delta$ at all points with $|\sigma| \leq e^{\delta \tau}$. After decreasing $\delta$, if necessary, we may assume that $C \delta$ is as small as we like. In particular, in the region we are considering, we always may assume that

$$
\left|u_{\sigma}\right| \leq \frac{1}{2}
$$

Since $v=u-1$, we immediately get estimates for $v$ and $v_{\sigma}$. Those for $v_{\sigma}$ are of course the same as for $u_{\sigma}$. For $v$, we have

$$
\begin{array}{r}
-\frac{C}{\tau} \leq v \leq C \frac{1+\sigma^{2}}{\tau} \quad \text { for }|\sigma| \leq 2 \sqrt{ } \tau \\
-\frac{C}{\tau} \leq v \leq C \frac{|\sigma|}{\sqrt{ } \tau} \sqrt{\log \frac{|\sigma|}{\sqrt{ } \tau}} \text { for } 2 \sqrt{ } \tau \leq|\sigma| \leq e^{\delta \tau}
\end{array}
$$

in which $C$ is again a constant depending only on $F^{*}$ and $n$.

Proof of Lemma 2.1. The upper estimates follow immediately from [3, Lemma 9.4]. Only the lower estimate in (2.9) was not proved in [3]. 
Briefly, the lower estimate follows from the boundedness of $F$. At the neck, one has

$$
K=\frac{\psi_{s s}}{\psi}, \quad L=\frac{1}{\psi^{2}}, \quad \psi=(1+\mathrm{o}(1)) \sqrt{2(n-1)(T-t)},
$$

such that $(K / L) \log L \leq C$ implies

$$
0 \leq \psi_{s s} \leq \frac{C}{\tau \psi}
$$

The radius $\psi(0, t)$ of the neck satisfies

$$
\psi_{t}=\psi_{s s}-\frac{n-1}{\psi} \leq-\frac{n-1-C\left(\tau^{-1}\right)}{\psi},
$$

which upon integration yields

$$
\psi(0, t) \geq\left(1-\frac{\tilde{C}}{\tau}\right) \sqrt{2(n-1)(T-t)},
$$

where $\tilde{C}$ only depends on $F^{*}$ and $n$. This implies the lower estimates in the lemma.

\subsection{Transition to commuting variables}

On most of the neck, the quantity $v=\mathrm{o}(1)$. So it is natural to drop the quadratic terms in (2.7), resulting in the deceptively simple equation

$$
v_{\tau}=v_{\sigma \sigma}+v
$$

for $v$. Here the partial derivatives $\partial_{\tau}$ and $\partial_{\sigma}$ do not commute. To work with commuting variables, we regard $v$ as function of $\sigma$. In other words, we define

$$
\sigma(x, t)=\frac{1}{\sqrt{T-t}} \int_{0}^{x} d s=e^{-\tau / 2} \int_{0}^{x} d s,
$$

and consider a function $\tilde{v}(\sigma, \tau)$ such that $\tilde{v}(\sigma(x, \tau), \tau) \equiv v(x, \tau)$. We shall abuse notation and write $v$ for both quantities $v$ and $\tilde{v}$. This is only ambiguous when we take time derivatives, in which case we write

$$
\left.\frac{\partial v}{\partial \tau}\right|_{\sigma \text { const }}=\frac{\partial \tilde{v}}{\partial \tau} \text { and }\left.\quad \frac{\partial v}{\partial \tau}\right|_{x \text { const }}=\frac{\partial v}{\partial \tau} .
$$


The two are related by

$$
\left.\frac{\partial v}{\partial \tau}\right|_{\sigma \text { const }}=\left.\frac{\partial v}{\partial \tau}\right|_{x \text { const }}-\frac{\partial \sigma}{\partial \tau} \frac{\partial v}{\partial \sigma}
$$

Lemma 2.2. Assuming reflection symmetry of the metric, i.e., $\psi(-s, t) \equiv$ $\psi(s, t)$, one has

$$
\frac{\partial \sigma}{\partial \tau}=\frac{\sigma}{2}+n J(\sigma, \tau)
$$

where

$$
J(\sigma, \tau)=\int_{0}^{\sigma} \frac{v_{\sigma \sigma}}{1+v} d \sigma=\frac{v_{\sigma}}{1+v}+\int_{0}^{\sigma} \frac{v_{\sigma}^{2}}{(1+v)^{2}} d \sigma
$$

Proof. By definition one has $\sigma(x, \tau)=e^{\tau / 2} s\left(x, T-e^{-\tau}\right)$. Hence

$$
\sigma_{\tau}=\frac{1}{2} \sigma+e^{\tau / 2} s_{t}\left(x, T-e^{-\tau}\right) e^{-\tau}=\frac{1}{2} \sigma+e^{-\tau / 2} s_{t}\left(x, T-e^{-\tau}\right) .
$$

One also has $[3, \S 10]$

$$
\frac{\partial s}{\partial t}=\int_{0}^{s} \frac{n \psi_{s s}}{\psi} d s=n\left\{\frac{\psi_{s}}{\psi}+\int_{0}^{s} \frac{\psi_{s}^{2}}{\psi^{2}} d s\right\} .
$$

Using the relations (2.2) and (2.3) between $u=1+v$ and $\psi$, and $s$ and $\sigma$, one finds the stated expression for $\partial_{\tau} \sigma$.

¿From here on we will consider $v$ as a function of $\sigma$ and $\tau$. All $\tau$ derivatives are intended to be time derivatives with $\sigma$ kept constant. It follows from (2.7) that $v$ satisfies

$$
v_{\tau}=v_{\sigma \sigma}-\frac{\sigma}{2} v_{\sigma}+v-n J(\sigma, \tau) v_{\sigma}+\frac{(n-1) v_{\sigma}^{2}-(1 / 2) v^{2}}{1+v}
$$

We write this equation as

$$
v_{\tau}=A(v)+N(v)
$$

where $A$ is the linear differential operator

$$
A=\left(\frac{\partial}{\partial \sigma}\right)^{2}-\frac{\sigma}{2} \frac{\partial}{\partial \sigma}+1
$$


and $N(v)$ represents the remaining (nonlinear) terms in (2.16),

$$
N(v)=-n v_{\sigma} \int_{0}^{\sigma} \frac{v_{\sigma \sigma}}{1+v} d \sigma+\frac{(n-1) v_{\sigma}^{2}-(1 / 2) v^{2}}{1+v} .
$$

Integrate by parts to get

$$
N(v)=-n v_{\sigma} \int_{0}^{\sigma} \frac{v_{\sigma}^{2}}{(1+v)^{2}} d \sigma-\frac{v_{\sigma}^{2}+(1 / 2) v^{2}}{1+v} .
$$

Lemma 2.3. For all $|\sigma| \leq e^{\delta \tau}$, one has

$$
|N(v)| \leq C \frac{1+\sigma^{4}}{\tau^{2}} .
$$

Proof. This follows from Lemma 2.1. In fact, Lemma 2.1 implies that

$$
|v| \leq C \frac{1+\sigma^{2}}{\tau} \text { and }\left|v_{\sigma}\right| \leq C \frac{1+|\sigma|}{\tau}
$$

for $|\sigma| \leq 2 \sqrt{ } \tau$. For larger $\sigma$, one finds other estimates for $v$ and $v_{\sigma}$, which are stronger than (2.18). Using (2.18) for all $|\sigma| \leq e^{\delta \tau}$, one arrives at our estimate for $N(v)$.

\subsection{The linearized equation}

If we ignore the nonlinear terms in (2.16), then we see that the small quantity $v$ satisfies $v_{\tau}=A(v)$. Even though $v$ is not defined for all $\sigma \in \mathbb{R}$, we know from our estimate on the neck that $v$ is defined for $|\sigma| \leq e^{\delta \tau}$, for some $\delta=\delta\left(F^{*}, n\right)$. In the first analysis, we assume that $v$ is, in fact, a solution of the Cauchy problem $v_{\tau}=A(v)$. The operator $A$ is self-adjoint in the Hilbert space

$$
\mathcal{H}=\left\{v \in L^{2}\left(\mathbb{R}, e^{-\sigma^{2} / 4} d \sigma\right) \mid v(\sigma) \equiv v(-\sigma)\right\} .
$$

It has a pure point spectrum, with eigenvalues $\lambda_{m}=1-m$ for $m=0,1,2, \ldots$. The eigenfunction corresponding to the eigenvalue $\lambda_{m}$ is an even Hermite polynomial of degree $2 \mathrm{~m}$. Normalizing the eigenfunction so that the coefficient of its highest order term is 1 , we set

$$
h_{2 m}(\sigma)=\sigma^{2 m}-\frac{(2 m) !}{1 !(2 m-2) !} \sigma^{2 m-2}+\frac{(2 m) !}{2 !(2 m-4) !} \sigma^{2 m-4}-\cdots .
$$


In particular,

$$
h_{0}(\sigma)=1, \quad h_{2}(\sigma)=\sigma^{2}-2, \quad h_{4}(\sigma)=\sigma^{4}-12 \sigma^{2}+12
$$

\subsection{Eigenfunction decomposition of $v$}

Let $\eta \in C^{\infty}(\mathbb{R})$ be an even bump function with $\eta(z)=0$ for $z \geq 1 \frac{1}{4}$ and $\eta(z)=1$ for $z \leq 1$. We then define

$$
V(\sigma, \tau)=\eta\left(\sigma e^{-\delta \tau / 2}\right) v(\sigma, \tau)
$$

For $|\sigma| \geq \frac{5}{4} e^{\delta \tau / 2}$, we set $V \equiv 0$. Then $V(\cdot, \tau) \in \mathcal{H}$, and $v \equiv V$ for $|\sigma| \leq e^{\delta \tau / 2}$.

A computation shows that

$$
V_{\tau}-A(V)=\eta N(v)+E,
$$

where the "error term" is

$$
E=\left(\eta_{\tau}-\eta_{\sigma \sigma}+\frac{\sigma}{2} \eta_{\sigma}\right) v-2 \eta_{\sigma} v_{\sigma}
$$

Lemma 2.4. The error term $E$ vanishes except when $e^{\delta \tau / 2} \leq|\sigma| \leq \frac{5}{4} e^{\delta \tau / 2}$. When $E \neq 0$, one has

$$
|E(\sigma, \tau)| \leq C|\sigma|
$$

where the constant $C$ only depends on $F^{*}$ and $n$. One also has

$$
\|E(\cdot, \tau)\| \leq C \exp \left(-e^{\delta \tau / 2}\right)
$$

where the constant $C$ again only depends on $F^{*}$ and $n$.

Proof. The pointwise estimate follows from the boundedness of $\eta_{\tau}, \sigma \eta_{\sigma}$ and $\eta_{\sigma \sigma}$, as well as the pointwise bounds for $v$ provided by Lemma 2.1. Given the pointwise bounds for $E$, one finds that

$$
\|E\|^{2} \leq C^{2} \int_{e^{\delta \tau / 2}}^{\infty} \sigma^{2} e^{-\sigma^{2} / 4} d \sigma
$$

from which the $\mathcal{H}$ norm estimate follows. 
For each $\tau$, the function $V(\cdot, \tau)$ belongs to $\mathcal{H}$. So we can consider the splitting into mutually orthogonal terms determined by

$$
V(\sigma, \tau)=a_{0}(\tau) h_{0}(\sigma)+a_{2}(\tau) h_{2}(\sigma)+W(\sigma, \tau)
$$

Our pointwise estimate (2.14) for $v$ suggests that for large $\tau$ these terms will decay like $\tau^{-1}$. This turns out to be the case for the middle term $a_{2}(\tau) h_{2}(\tau)$, but the other two terms are in fact smaller. We will show in the next few paragraphs that they decay like $\tau^{-2}$. This establishes $a_{2}(\tau) h_{2}(\tau)$ as the dominant term for large $\tau$.

\subsection{Easy estimates for $a_{0}$ and $a_{2}$}

Lemma 2.1 and in particular (2.14) imply that

$$
|V(\sigma, \tau)| \leq \frac{C}{\tau}\left(1+\sigma^{2}\right)
$$

for all $\sigma \in \mathbb{R}$, and all $\tau \geq \tau_{0}$. Upon taking the $\mathcal{H}$-inner product with $h_{0}$ and $h_{2}$ this gives us

$$
\left|a_{0}(\tau)\right|+\left|a_{2}(\tau)\right| \leq \frac{C}{\tau}
$$

for all $\tau \geq \tau_{0}$.

\subsection{Decay of $a_{0}$}

Since $\left\|h_{0}\right\|^{2} a_{0}(\tau)=\left(h_{0}, V\right)_{\mathcal{H}}$, we have

$$
\begin{aligned}
\left\|h_{0}\right\|^{2} a_{0}^{\prime}(\tau) & =\left(h_{0}, V_{\tau}\right)_{\mathcal{H}} \\
& =\left(h_{0}, A(V)+\eta N(v)+E\right)_{\mathcal{H}} \\
& =\left(h_{0}, V\right)_{\mathcal{H}}+\left(h_{0}, \eta N(v)+E\right)_{\mathcal{H}} \\
& =\left\|h_{0}\right\|^{2} a_{0}(\tau)+\left(h_{0}, \eta N(v)+E\right)_{\mathcal{H}} .
\end{aligned}
$$

Hence

$$
a_{0}^{\prime}(\tau)-a_{0}(\tau)=\left(\frac{h_{0}}{\left\|h_{0}\right\|^{2}}, \eta N(v)+E\right)_{\mathcal{H}} .
$$

In other words, $a_{0}^{\prime}(\tau)=a_{0}(\tau)$ plus a small error term. If this error term were absent, we would have $a_{0}^{\prime}(\tau)=a_{0}(\tau)$, and the only possible solution 
with $a_{0}(\infty)=0$ would be $a_{0}(0) \equiv 0$. The presence of the error term causes $a_{0}(\tau)$ to deviate from zero, and we now proceed to estimate this deviation.

Using our estimates for $N(v)$ and $E$, we conclude that

$$
f_{0}(\tau) \doteqdot a_{0}^{\prime}(\tau)-a_{0}(\tau)
$$

satisfies

$$
\left|f_{0}(\tau)\right| \leq \frac{C\left(F^{*}, n\right)}{\tau^{2}}
$$

The variation of constants formula tells us that

$$
a_{0}(\tau)=e^{\tau-\tau_{1}} a_{0}\left(\tau_{1}\right)-\int_{\tau}^{\tau_{1}} e^{\tau-\tau^{\prime}} f_{0}\left(\tau^{\prime}\right) d \tau^{\prime},
$$

in which we can let $\tau_{1} \rightarrow \infty$, to get

$$
a_{0}(\tau)=-\int_{\tau}^{\infty} e^{\tau-\tau^{\prime}} f_{0}\left(\tau^{\prime}\right) d \tau^{\prime}
$$

Hence

$$
\left|a_{0}(\tau)\right| \leq \frac{C\left(F^{*}, n\right)}{\tau^{2}} .
$$

\subsection{Decay of $W$}

Since $W$ lies in the stable space of the operator $A$, i.e., the space spanned by those eigenfunctions with negative eigenvalues, we expect $W$ to decay according to the slowest stable eigenvalue (i.e., $\sim e^{-\tau}$ ), or else to decay like the "forcing term" $\eta N(v)+E$. In this situation, the forcing term is dominant, and we have the following.

Lemma 2.5. For any given $\tau_{0}>0$, one has for all $\tau>\tau_{0}$

$$
\|W(\cdot, \tau)\| \leq e^{\tau_{0}-\tau}\left\|W\left(\cdot, \tau_{0}\right)\right\|+\frac{C}{\tau^{2}} .
$$

Consequently, one also has

$$
\|W(\cdot, \tau)\| \leq \frac{C}{\tau^{2}}\left(1+W_{0}\right) .
$$

Here the constant $C$ only depends on $F^{*}$ and $n$, and

$$
W_{0} \doteqdot e^{\tau_{0}}\left\|W\left(\cdot, \tau_{0}\right)\right\|
$$


Proof. Since $\|W\|^{2}=(W, W)=(W, V)$ and $(W, A(V))_{\mathcal{H}}=(W, A(W))_{\mathcal{H}}$, one has

$$
\begin{aligned}
\|W\| \frac{d\|W\|}{d \tau} & =\frac{1}{2} \frac{d}{d \tau}\|W\|^{2} \\
& =\frac{1}{2} \frac{d}{d \tau}(W, V)_{\mathcal{H}} \\
& =\left(W, V_{\tau}\right)_{\mathcal{H}} \\
& =(W, A(V)+\eta N(v)+E)_{\mathcal{H}} \\
& =(W, A(W))_{\mathcal{H}}+(W, \eta N(v)+E)_{\mathcal{H}} \\
& \leq-\|W\|^{2}+\|W\| \cdot\|\eta N(v)+E\|,
\end{aligned}
$$

whence

$$
\frac{d\|W\|}{d \tau} \leq-\|W\|+\|\eta N(v)+E\| .
$$

¿From Lemma 2.3 we conclude that $|\eta N(v)| \leq C\left(1+\sigma^{4}\right) \tau^{-2}$ for all $\sigma$, and hence that

$$
\|\eta N(v)\| \leq \frac{C}{\tau^{2}} .
$$

For $E$, we use Lemma 2.4 together with the calculus inequality $C e^{-e^{\delta \tau / 2}} \leq$ $C^{\prime} \tau^{-2}$. We apply all this to (2.24) and conclude that

$$
\frac{d\|W\|}{d \tau} \leq-\|W\|+\frac{C}{\tau^{2}} .
$$

Therefore

$$
\frac{d\left(e^{\tau}\|W\|\right)}{d \tau} \leq C e^{\tau} \tau^{-2}
$$

Integration leads to

$$
e^{\tau}\|W(\cdot, \tau)\| \leq e^{\tau_{0}}\left\|W\left(\cdot, \tau_{0}\right)\right\|+C \int_{\tau_{0}}^{\tau} \frac{e^{\tilde{\tau}}}{\tilde{\tau}^{2}} d \tilde{\tau} \leq e^{\tau_{0}}\left\|W\left(\cdot, \tau_{0}\right)\right\|+\frac{\tilde{C}}{\tau^{2}} e^{\tau},
$$

as claimed. The second estimate for $\|W\|$ follows directly from $\sup \tau^{2} e^{-\tau}<\infty$.

\subsection{Derivative estimates for $W$}

We use the regularizing effect of the heat equation to bootstrap the estimates of Lemma 2.5 by one-space derivative. 
Lemma 2.6. Let $\tau_{0}>0$ be as in Lemma 2.5. Then for all $\tau>\tau_{0}+1$, one has

$$
\left\|W_{\sigma}(\cdot, \tau)\right\| \leq C\left(1+W_{0}\right) \tau^{-2}
$$

where the constant $C$ only depends on $F^{*}$ and $n$, and as before, $W_{0}=$ $e^{\tau_{0}}\left\|W\left(\cdot, \tau_{0}\right)\right\|$

Proof. Note that

$$
A u=u_{\sigma \sigma}-\frac{1}{2} \sigma u_{\sigma}+u=e^{\sigma^{2} / 4}\left(e^{-\sigma^{2} / 4} u_{\sigma}\right)_{\sigma}
$$

so that for $u \in D(A)$ one has

$$
-(u, A u)_{\mathcal{H}}=\int_{\mathbb{R}} e^{-\sigma^{2} / 4}\left\{u_{\sigma}^{2}-u^{2}\right\} d \sigma .
$$

Thus

$$
\left\|\partial_{\sigma} u\right\|^{2}=\|u\|^{2}-(u, A u)
$$

If $u \perp h_{0}, h_{2}$ then $(u,-A u)_{\mathcal{H}} \geq 0$, and we have

$$
\left\|\partial_{\sigma} u\right\| \leq\|u\|+\sqrt{(u,-A u)_{\mathcal{H}}} .
$$

If we substitute $e^{\theta A} u$ for $u$ in this inequality then we get

$$
\left\|\partial_{\sigma} e^{\theta A} u\right\| \leq \frac{C}{\sqrt{ } \theta}\|u\|
$$

for $0<\theta \leq 1$, and all $u \in D(A)$. Indeed, expand $u$ in eigenvectors of $A$,

$$
u=\sum_{k=2}^{\infty} u_{k} \hat{h}_{2 k}, \quad \hat{h}_{2 k} \doteqdot \frac{h_{2 k}}{\left\|h_{2 k}\right\|} .
$$

Then, with $\lambda_{k}=1-k$,

$$
\begin{aligned}
\left(e^{\theta A} u,-A e^{\theta A} u\right) & =\sum_{k \geq 2} \lambda_{k} e^{-2 \theta \lambda_{k}}\left|u_{k}\right|^{2} \\
& \leq\left(\sup _{k \geq 2} \lambda_{k} e^{-2 \theta \lambda_{k}}\right) \sum_{k \geq 2}\left|u_{k}\right|^{2}
\end{aligned}
$$




$$
\begin{aligned}
& \leq\left(\sup _{\lambda>0} \lambda e^{-2 \theta \lambda}\right)\|u\|^{2} \\
& =\frac{C}{\theta}\|u\|^{2}
\end{aligned}
$$

from which (2.26) follows.

The stable component $W$ of $V$ satisfies

$$
W_{\tau}=A(W)+f(\tau), \quad \text { where } f(\tau) \doteqdot \mathcal{P}(\eta N(v)+E),
$$

where $\mathcal{P}$ is the $\mathcal{H}$-orthogonal projection onto the space $\left\{u \in \mathcal{H} \mid u \perp h_{0}, h_{2}\right\}$. By the variation of constants formula one then has, for all $\tau \geq \tau_{0}+1$

$$
W(\tau)=e^{A} W(\tau-1)+\int_{0}^{1} e^{\theta A} f(\tau-\theta) d \theta .
$$

Since $f(\tau) \perp\left\{h_{0}, h_{2}\right\}$, we can use (2.26) to get

$$
\begin{aligned}
\left\|\partial_{\sigma} W(\tau)\right\| & \leq\left\|\partial_{\sigma} e^{A} W(\tau-1)\right\|+\int_{0}^{1}\left\|\partial_{\sigma} e^{\theta A} f(\tau-\theta)\right\| d \theta \\
& \leq C\|W(\tau-1)\|+\int_{0}^{1} \frac{C}{\sqrt{ } \theta}\|f(\tau-\theta)\| d \theta .
\end{aligned}
$$

The combination of Lemmas 2.3 and 2.4 again gives $\|f(\tau)\| \leq C \tau^{-2}$. The integral can therefore be bounded by $C \tau^{-2}$. Combining this with our previous estimate for $\|W(\tau-1)\|$ from Lemma 2.5, we get the inequality in Lemma 2.6.

\subsection{A bound for $\|\sigma W\|$}

If $\varphi \in C_{c}^{1}(\mathbb{R})$, then one has the identity

$$
\int_{\mathbb{R}} e^{-\sigma^{2} / 4} 2 \varphi(\sigma) \sigma \varphi^{\prime}(\sigma) d \sigma=\int_{\mathbb{R}} e^{-\sigma^{2} / 4}\left(-2 \varphi(\sigma)^{2}+\frac{1}{2} \sigma^{2} \varphi(\sigma)^{2}\right) d \sigma
$$

which after rearranging leads to

$$
\begin{aligned}
\|\sigma \varphi\|^{2} & =4 \int_{R} e^{-\sigma^{2} / 4}\left(\sigma \varphi \varphi^{\prime}+\varphi^{2}\right) d \sigma \\
& =4\left\{\left(\sigma \varphi, \varphi^{\prime}\right)_{\mathcal{H}}+\|\varphi\|^{2}\right\} \\
& \leq \frac{1}{2}\|\sigma \varphi\|^{2}+8\left\|\varphi^{\prime}\right\|^{2}+4\|\varphi\|^{2} \\
& \leq 16\left\{\left\|\varphi^{\prime}\right\|^{2}+\|\varphi\|^{2}\right\} .
\end{aligned}
$$


An approximation argument leads to

Lemma 2.7. For any $\varphi \in \mathcal{H}$ with $\varphi_{\sigma} \in \mathcal{H}$ one has $\sigma \varphi \in \mathcal{H}$, while

$$
\|\sigma \varphi(\sigma)\| \leq 4\left\{\left\|\varphi_{\sigma}\right\|+\|\varphi\|\right\}
$$

In particular, $W$ satisfies

$$
\|\sigma W\| \leq C\left(1+W_{0}\right) \tau^{-2}
$$

for all $\tau \geq \tau_{0}+1$.

\subsection{Pointwise estimates for $W$}

A Sobolev inequality says that the bound on $\left\|\partial_{\sigma} W\right\|$ implies a pointwise bound for $W$. Due to the exponential weight in the norm this bound is not uniform. One has

Lemma 2.8. Any $\varphi \in \mathcal{H}$ with $\partial_{\sigma} \varphi \in \mathcal{H}$ is a continuous function, which satisfies

$$
e^{-\sigma^{2} / 8}|\varphi(\sigma)| \leq C\left(\|\varphi\|+\left\|\partial_{\sigma} \varphi\right\|\right)
$$

Hence $W$ satisfies

$$
|W(\sigma, \tau)| \leq \frac{C\left(1+W_{0}\right)}{\tau^{2}} e^{\sigma^{2} / 8}
$$

Proof. Assuming $\varphi \in C_{c}^{1}(\mathbb{R})$ one has

$$
\begin{aligned}
e^{-\sigma^{2} / 4} \varphi(\sigma)^{2} & =\int_{-\infty}^{\sigma} e^{-\sigma^{2} / 4}\left[-\frac{\sigma}{2} \varphi^{2}+2 \varphi \varphi_{\sigma}\right] d \sigma \\
& \leq\|\sigma \varphi\|\|\varphi\|+\|\varphi\|\left\|\varphi_{\sigma}\right\| \\
& \leq C\left\{\|\varphi\|^{2}+\left\|\varphi_{\sigma}\right\|^{2}\right\} .
\end{aligned}
$$

So the pointwise estimate holds for $\varphi \in C_{c}^{1}$. By approximation it also holds for all $\varphi \in \mathcal{H}$ with $\partial_{\sigma} \varphi \in \mathcal{H}$.

Lemma 2.8 says that $W$ decays pointwise like $\tau^{-2}$, and uniformly on bounded $\sigma$ intervals. The following pointwise bound for $W$ only gives a $\tau^{-1}$ decay rate, but it is stronger for large values of $\sigma$. The estimate also does not depend on $W_{0}$. 
Lemma 2.9. For some constant $C=C\left(F^{*}, n\right)<\infty$ one has for all $\tau>$ $\tau_{0}+1$

$$
|W(\sigma, \tau)| \leq \frac{C}{\tau}\left(1+\sigma^{2}\right)
$$

Proof. We have, by definition, $W(\sigma, \tau)=V(\sigma, \tau)-a_{0}(\tau) h_{0}(\sigma)-a_{2}(\tau) h_{2}(\sigma)$. Using our pointwise bounds for $V, a_{0}, a_{2}$, and $h_{0}(\sigma) \equiv 1, h_{2}(\sigma) \equiv \sigma^{2}-2$, one quickly gets the stated estimate for $W$.

\subsection{Pointwise estimate for $W_{\sigma}$}

By definition we have $W(\tau, \sigma)=V(\tau, \sigma)-a_{0}(\tau) h_{0}(\sigma)-a_{2}(\tau) h_{2}(\sigma)$. Differentiate and keep in mind that $h_{0}^{\prime}(\sigma)=0$ and $h_{2}^{\prime}(\sigma)=2 \sigma$. We get $W_{\sigma}=$ $V_{\sigma}-2 a_{2}(\tau) \sigma$. Using the pointwise derivative bounds (2.11) and (2.12) for $u$, one then gets

$$
\left|W_{\sigma}\right| \leq \frac{C}{\tau}(1+|\sigma|)
$$

which holds for all $\tau \geq \tau_{0}$ and $\sigma \in \mathbb{R}$.

\subsection{An equation for $a_{2}$}

As with $a_{0}$, we have

$$
\begin{aligned}
\left\|h_{2}\right\|^{2} a_{2}^{\prime}(\tau) & =\left(h_{2}, V_{\tau}\right)_{\mathcal{H}} \\
& =\left(h_{2}, A(V)+\eta N(v)+E\right)_{\mathcal{H}} \\
& =\left(A\left(h_{2}\right), V\right)_{\mathcal{H}}+\left(h_{2}, \eta N(v)+E\right)_{\mathcal{H}} \\
& =\left(h_{2}, \eta N(v)+E\right)_{\mathcal{H}} .
\end{aligned}
$$

We use $(2.17)$ and the identity $1 /(1+v)=1-v /(1+v)$ to rewrite $N(v)$ as $(2.29)$

$$
N(v)=N_{2}(v)+N_{3}(v), \quad \text { where }\left\{\begin{array}{c}
N_{2}(v)=-\left(v_{\sigma}^{2}+\frac{1}{2} v^{2}\right) \\
N_{3}(v)=-n v_{\sigma} \int_{0}^{\sigma} \frac{v_{\sigma}^{2}}{(1+v)^{2}} d \sigma \\
+\frac{v v_{\sigma}^{2}+(1 / 2) v^{3}}{1+v}
\end{array}\right.
$$

Here, $N_{2}(v)$ is the purely quadratic part, while $N_{3}(v)$ contains the cubic and higher order terms. 
Lemma 2.10. There is a constant $C=C\left(F^{*}, n\right)$ such that for all $\tau \geq \tau_{0}$

$$
\left\|\eta N_{3}(v)\right\| \leq \frac{C}{\tau^{3}}
$$

Proof. The pointwise estimates for $v$ and $v_{\sigma}$ from (2.14) imply that

$$
|v| \leq C \frac{1+\sigma^{2}}{\tau}, \quad\left|v_{\sigma}\right| \leq C \frac{1+|\sigma|}{\tau}
$$

whenever $|\sigma| \leq e^{\delta \tau}$. Hence we have

$$
\left|N_{3}(v)\right| \leq C \frac{1+\sigma^{6}}{\tau^{3}} \quad \text { for }|\sigma| \leq e^{\delta \tau}
$$

(The term with $v^{3}$ contributes the highest power in $\sigma$.)

This implies the lemma.

We continue with our computation of $a_{2}^{\prime}(\tau)$. We have

$$
\begin{aligned}
\left\|h_{2}\right\|^{2} a_{2}^{\prime}(\tau) & =\left(h_{2}, \eta N(v)+E\right)_{\mathcal{H}} \\
& =\left(h_{2}, \eta N_{2}(v)+\eta N_{3}(v)+E\right)_{\mathcal{H}} \\
& =\left(h_{2}, \eta N_{2}(v)\right)_{\mathcal{H}}+\left(h_{2}, \eta N_{3}(v)+E\right)_{\mathcal{H}} .
\end{aligned}
$$

The last term satisfies

$$
\left|\left(h_{2}, \eta N_{3}(v)+E\right)_{\mathcal{H}}\right| \leq\left\|h_{2}\right\|\left(\left\|\eta N_{3}(v)\right\|+\|E\|\right) \leq \frac{C}{\tau^{3}}
$$

We would now like to replace the $v$ in the quadratic expression $N_{2}(v)$ by $V$, and then by the dominant term in $(2.22)$, i.e., $a_{2}(\tau) h_{2}(\sigma)$. Before we do this, we estimate the errors produced by these replacements.

Lemma 2.11. There is a constant $C=C\left(F^{*}, n\right)$ such that for all $\tau \geq \tau_{0}$, one has

$$
\left\|\eta\left(N_{2}(v)-N_{2}(V)\right)\right\| \leq C e^{-e^{\delta \tau / 2}}
$$

In other words $\eta\left(N_{2}(v)-N_{2}(V)\right)$ satisfies the same super-exponentially small estimate as the error term $E$; see Lemma 2.4 . 
Proof. Since $V=\eta \cdot v$, a direct calculation gives

$$
\eta\left(N_{2}(v)-N_{2}(V)\right)=\eta\left\{(\eta v)_{\sigma}^{2}-v_{\sigma}^{2}\right\}+\frac{1}{2} \eta\left(\eta^{2}-1\right) v^{2} .
$$

Using the pointwise estimates for $v$ and $v_{\sigma}$ with the fact that $\eta\left(N_{2}(v)-N_{2}(V)\right)$ is supported in the region $e^{\delta \tau / 2} \leq|\sigma| \leq \frac{5}{4} e^{\delta \tau / 2}$, one arrives at the stated estimate for the $\mathcal{H}$ norm of $\eta\left(N_{2}(v)-N_{2}(V)\right)$.

We therefore have

$$
\begin{aligned}
& \left\|h_{2}\right\|_{\mathcal{H}}^{2} a_{2}^{\prime}(\tau)=\left(h_{2}, \eta N_{2}(V)\right)_{\mathcal{H}}+\left(h_{2}, \eta\left(N_{2}(v)-N_{2}(V)\right)\right)_{\mathcal{H}} \\
& \quad+\left(h_{2}, \eta N_{3}(v)+E\right)_{\mathcal{H}} .
\end{aligned}
$$

If we write $N_{2}[v, w]=-v_{\sigma} w_{\sigma}-\frac{1}{2} v w$ for the natural symmetric bilinear expression with $N_{2}(v)=N_{2}[v, v]$, then the first term above can be written as

$$
\begin{aligned}
\left(h_{2}, \eta N_{2}(V)\right)_{\mathcal{H}}= & \left(\eta h_{2}, N_{2}\left(a_{2} h_{2}+a_{0} h_{0}+W\right)\right)_{\mathcal{H}} \\
= & \left(a_{2}\right)^{2}\left(\eta h_{2}, N_{2}\left(h_{2}\right)\right)_{\mathcal{H}} \\
& +2 a_{2}\left(\eta h_{2}, N_{2}\left[h_{2}, a_{0} h_{0}+W\right]\right)_{\mathcal{H}} \\
& +\left(\eta h_{2}, N_{2}\left(a_{0} h_{0}+W\right)\right)_{\mathcal{H}},
\end{aligned}
$$

which is a quadratic polynomial in $a_{2}$. Adding in the omitted error terms, we reach the following observation.

\section{Lemma 2.12.}

$$
a_{2}^{\prime}(\tau)=K(\tau) a_{2}(\tau)^{2}+2 L(\tau) a_{2}(\tau)+M(\tau)
$$

in which

$$
\begin{aligned}
K(\tau)= & \frac{1}{\left\|h_{2}\right\|^{2}}\left(\eta h_{2}, N_{2}\left(h_{2}\right)\right)_{\mathcal{H}}, \\
L(\tau)= & \frac{1}{\left\|h_{2}\right\|^{2}}\left(\eta h_{2}, N_{2}\left[h_{2}, a_{0} h_{0}+W\right]\right)_{\mathcal{H}}, \\
M(\tau)= & \frac{1}{\left\|h_{2}\right\|^{2}}\left\{\left(\eta h_{2}, N_{2}\left(a_{0} h_{0}+W\right)\right)_{\mathcal{H}}+\left(h_{2}, \eta\left(N_{2}(v)-N_{2}(V)\right)\right)_{\mathcal{H}}\right. \\
& \left.+\left(h_{2}, \eta N_{3}(v)+E\right)_{\mathcal{H}}\right\} .
\end{aligned}
$$

\section{Lemma 2.13.}

$$
K(\tau)=-8+\mathcal{O}\left(e^{-e^{\delta \tau / 2}}\right)
$$


Proof. Since $N_{2}(v)=-v_{\sigma}^{2}-\frac{1}{2} v^{2}$, the explicit expressions (2.19) for $h_{0}, h_{2}$, and $h_{4}$ give us

$$
N_{2}\left(h_{2}\right)=-\frac{1}{2} \sigma^{4}-2 \sigma^{2}-2=-\frac{1}{2} h_{4}-8 h_{2}-12 h_{0} .
$$

Hence $\left(h_{2}, N_{2}\left(h_{2}\right)\right)_{\mathcal{H}}=-8\left\|h_{2}\right\|^{2}$. This implies

$$
K(\tau)=\frac{\left(h_{2}, N_{2}\left(h_{2}\right)\right)_{\mathcal{H}}}{\left\|h_{2}\right\|^{2}}+\frac{\left((1-\eta) h_{2}, N_{2}\left(h_{2}\right)\right)_{\mathcal{H}}}{\left\|h_{2}\right\|^{2}} .
$$

The first term is -8 . The other term can be written as the integral of a function with fixed polynomial growth, which is supported in the region $|\sigma| \geq e^{\delta \tau / 2}$. This leads quickly to the stated estimate.

Lemma 2.14. $L(\tau)=\mathcal{O}\left(\tau^{-2}\right)$, i.e., there is a constant $C=C\left(F^{*}, n\right)$ such that for all $\tau \geq \tau_{0}+1$ one has $\left|\tau^{2} L(\tau)\right| \leq C$.

Proof. Using (bi)linearity of $N_{2}[v, \tilde{v}]$, we get

$$
\begin{aligned}
\left\|h_{2}\right\|^{2} L(\tau) & =\left(\eta h_{2}, N_{2}\left[h_{2}, a_{0} h_{0}+W\right]\right)_{\mathcal{H}} \\
& =a_{0}(\tau)\left(h_{2}, \eta N_{2}\left(h_{2}, h_{0}\right)\right)_{\mathcal{H}}+\left(h_{2}, \eta N_{2}\left(h_{2}, W\right)\right)_{\mathcal{H}} .
\end{aligned}
$$

The first term is $\mathcal{O}\left(\tau^{-2}\right)$. For the other term, we have

$$
\begin{aligned}
\left|\left(h_{2}, \eta N_{2}\left(h_{2}, W\right)\right)_{\mathcal{H}}\right| & =\left|\left(\eta h_{2}, h_{2}^{\prime}(\sigma) W_{\sigma}+\frac{1}{2} h_{2} W\right)_{\mathcal{H}}\right| \\
& \leq\left\|\eta h_{2} h_{2}^{\prime}\right\|\left\|W_{\sigma}\right\|+C\|W\| \\
& \leq \frac{C}{\tau^{2}}
\end{aligned}
$$

by our estimates for $\|W\|$ and $\left\|\partial_{\sigma} W\right\|$.

Lemma 2.15.

$$
M(\tau) \leq C\left\{\frac{e^{\tau_{0}-\tau}}{\tau}\left\|W\left(\cdot, \tau_{0}\right)\right\|+\frac{1}{\tau^{3}}\right\}
$$

In fact, one can show that $M(\tau) \leq C_{\alpha} \tau^{-(4-\alpha)}$ for any $\alpha>0$, but we will only need the estimate with $\alpha=1$.

Proof. In Lemma 2.12, $\left\|h_{2}\right\|^{2} M(\tau)$ is defined as the sum of three terms. We now estimate them one by one. 
The middle term is

$$
\left|\left(h_{2}, \eta\left(N_{2}(v)-N_{2}(V)\right)\right)_{\mathcal{H}}\right| \leq\left\|h_{2}\right\|\left\|\eta\left(N_{2}(v)-N_{2}(V)\right)\right\| \leq C e^{-e^{\delta \tau / 2}}
$$

by Lemma 2.11 .

The third term is bounded by

$$
\left|\left(h_{2}, \eta N_{3}(v)+E\right)_{\mathcal{H}}\right| \leq C\left(\left\|\eta N_{3}(v)\right\|+\|E\|\right) \leq \frac{C}{\tau^{3}},
$$

because of Lemma 2.10 and the super-exponential estimate for $E$ in Lemma 2.4.

The first term can itself be split into three terms:

$$
\begin{aligned}
& \left(\eta h_{2}, N_{2}\left(a_{0} h_{2}+W\right)\right)_{\mathcal{H}} \\
& \quad=a_{0}(\tau)^{2}\left(\eta h_{2}, N_{2}\left(h_{2}\right)\right)_{\mathcal{H}}+2 a_{0}(\tau)\left(\eta h_{2}, N_{2}\left[h_{2}, W\right]\right)_{\mathcal{H}}+\left(\eta h_{2}, N_{2}(W)\right)_{\mathcal{H}} .
\end{aligned}
$$

The first of these terms is $\mathcal{O}\left(\tau^{-4}\right)$, since $a_{0}(\tau)=\mathcal{O}\left(\tau^{-2}\right)$.

From

$$
\begin{aligned}
\left(\eta h_{2}, N_{2}\left[h_{2}, W\right]\right)_{\mathcal{H}} & =\left(\eta h_{2},-h_{2}^{\prime}(\sigma) W_{\sigma}-\frac{1}{2} h_{2} W\right)_{\mathcal{H}} \\
& =-\left(\eta h_{2} h_{2}^{\prime}, W_{\sigma}\right)_{\mathcal{H}}-\frac{1}{2}\left(\eta h_{2}(\sigma)^{2}, W\right)_{\mathcal{H}} \\
& =\mathcal{O}\left(\left\|W_{\sigma}\right\|+\|W\|\right) \\
& =\mathcal{O}\left(\tau^{-2}\right)
\end{aligned}
$$

and $a_{0}(\tau)=\mathcal{O}\left(\tau^{-2}\right)$, we deduce that the middle term in $(2.30)$ is also $\mathcal{O}\left(\tau^{-4}\right)$.

We are left with the last term in (2.30). This term can be expanded as

$$
\left(\eta h_{2}, N_{2}(W)\right)_{\mathcal{H}}=-\left(\eta h_{2}, W_{\sigma}^{2}\right)_{\mathcal{H}}-\left(\eta h_{2}, \frac{1}{2} W^{2}\right)_{\mathcal{H}} .
$$

The second term satisfies

$$
\begin{aligned}
\left|\int_{\mathbb{R}} e^{-\sigma^{2} / 4} \eta h_{2}(\sigma) W(\tau, \sigma)^{2} d \sigma\right| & \leq C\left(\|W\|^{2}+\|\sigma W\|^{2}\right) \\
& \leq C\left\{e^{2\left(\tau_{0}-\tau\right)}\left\|W\left(\cdot, \tau_{0}\right)\right\|^{2}+\frac{1}{\tau^{4}}\right\}
\end{aligned}
$$

by Lemmas 2.5 and 2.7 . 
We bound the first term in (2.31) as follows:

$$
\begin{aligned}
\left|\left(\eta h_{2}, W_{\sigma}^{2}\right)_{\mathcal{H}}\right| & =\left|\int_{\mathbb{R}} e^{-\sigma^{2} / 4} \eta h_{2}(\sigma) W_{\sigma}^{2} d \sigma\right| \\
& \leq C\left|\int_{\mathbb{R}} e^{-\sigma^{2} / 4}\left(1+\sigma^{2}\right) W_{\sigma}^{2} d \sigma\right| \\
& \leq C\left\|(1+\sigma)^{2} W_{\sigma}\right\|\left\|W_{\sigma}\right\| .
\end{aligned}
$$

The pointwise estimate (2.28) bounds the first factor by

$$
\left\|(1+\sigma)^{2} W_{\sigma}\right\| \leq \frac{C}{\tau}
$$

while Lemma 2.6 implies

$$
\left\|W_{\sigma}\right\| \leq C\left\{e^{\tau_{0}-\tau}\left\|W\left(\cdot, \tau_{0}\right)\right\|+\frac{1}{\tau^{2}}\right\} .
$$

Combined, these last two estimates imply Lemma 2.15.

\subsection{Dichotomy for the decay of $a_{2}(\tau)$}

We have shown that

$$
\frac{d a_{2}(\tau)}{d \tau}=-8 a_{2}(\tau)^{2}+M^{*}(\tau)
$$

where

$$
\left|M^{*}(\tau)\right| \leq C\left(1+W_{0}\right) \tau^{-3}
$$

Hence

$$
\tau \frac{d\left(\tau a_{2}(\tau)\right)}{d \tau}=\left(\tau a_{2}(\tau)\right)-8\left(\tau a_{2}(\tau)\right)^{2}+\tau^{2} M^{*}(\tau)
$$

Lemma 2.16. The coefficient $a_{2}(\tau)$ satisfies either

$$
a_{2}(\tau) \leq C\left(1+W_{0}\right) \tau^{-2}
$$

for all $\tau \geq \tau_{0}+1$, or else

$$
\lim _{\tau \rightarrow \infty} \tau a_{2}(\tau)=\frac{1}{8}
$$




\subsection{Pinching time}

We will show below that alternative (2.34) does indeed occur, namely that there exists an open set of solutions matching the asymptotic profile we have constructed. Our first step is to improve the estimate obtained in [3, Lemma 6.1 . Let $r(t) \equiv \psi(0, t)$ denote the radius of the neck, and recall that

$$
K=\frac{\psi_{s s}}{\psi} \quad \text { and } \quad L=\frac{1-\psi_{s}^{2}}{\psi^{2}}
$$

denote the sectional curvatures. Define $\lambda=2-\log L_{\min }(0)$, so that we may write the cylindricality $F$ as

$$
F=\frac{K}{L}(\lambda+\log L)=\frac{\psi \psi_{s s}}{1-\psi_{s}^{2}}\left(\lambda+\log \frac{1-\psi_{s}^{2}}{\psi^{2}}\right) .
$$

Recall that there exists an $F^{*} \geq n-1$ depending only on $g(0)$ such that $0 \leq F \leq F^{*}$ on the neck. Writing the evolution equation for the radius

$$
\frac{d r}{d t}=\left[\psi_{s s}-(n-1) \frac{1-\psi_{s}^{2}}{\psi}\right]_{s=0}=\psi_{s s}-\frac{n-1}{r}
$$

in the form

$$
\frac{d}{d t}\left(r^{2}\right)=-2(n-1)+2 \frac{F}{\lambda+\log 1 / r^{2}}
$$

then proves that

$$
-2(n-1) \leq \frac{d}{d t}\left(r^{2}\right) \leq-2(n-1)+2 \frac{F^{*}}{\lambda+\log 1 / r^{2}} .
$$

This observation allows us to prove that the singularity time depends continuously on the initial conditions.

Lemma 2.17. For rotationally symmetric metrics $g(t)$ that develop a neckpinch, the pinching time $T$ is a continuous function of the initial metric $g_{0}$.

Proof. Given a metric $g_{0}$, let $T_{0}=T\left(g_{0}\right)$ denote the singularity time of the solution $g(t)$ with initial data $g_{0}$.

To prove lower semicontinuity, let $T_{-}<T_{0}$ be given. Then $\{g(t): 0 \leq$ $\left.t \leq T_{-}\right\}$is a smooth solution of Ricci flow. Because regular solutions of parabolic equations depend continuously on their initial data, a solution 
$\tilde{g}(t)$ will remain smooth for $0 \leq t \leq T_{-}$, provided that its initial data $\tilde{g}_{0}$ is sufficiently close to $g_{0}$. This implies that the singularity time $\tilde{T}$ of $\tilde{g}(\cdot)$ satisfies $\tilde{T}>T_{-}$.

To prove upper semicontinuity, let $T_{+}>T_{0}$ be given. At any time $T_{*}<$ $T_{0}$, observe that for all $\tilde{g}_{0}$ sufficiently close to $g_{0}$, the radius $\tilde{r}$ of the solution $\tilde{g}(t)$ satisfies $\tilde{r}\left(T_{*}\right)^{2} \leq 2 r\left(T_{*}\right)^{2}$, and its cylindricality $\tilde{F}$ satisfies $\tilde{F} \leq \tilde{F}^{*} \leq$ $2 F^{*}$. By taking $T_{*}$ close enough to $T_{0}$, one may by $(2.36)$ ensure that any solution $\tilde{g}(t)$ with initial data $\tilde{g}_{0}$ satisfies

$$
\frac{d}{d t}\left(\tilde{r}^{2}\right) \leq-2(n-1)+\frac{4 F^{*}}{\lambda+\log 1 / 2 r\left(T_{*}\right)^{2}} \leq-(n-1)
$$

for all $t \in\left(T_{*}, \tilde{T}\right)$, where $\tilde{T}$ is the pinching time of $\tilde{g}(t)$. So for $t \in\left(T_{*}, \tilde{T}\right)$, it follows that

$$
\begin{aligned}
\tilde{r}(t)^{2} & \leq \tilde{r}\left(T_{*}\right)^{2}-(n-1)\left(t-T_{*}\right) \\
& \leq 2 r\left(T_{*}\right)^{2}-(n-1)\left(t-T_{*}\right) \\
& \leq 4(n-1)\left(T_{0}-T_{*}\right)-(n-1)\left(t-T_{*}\right) .
\end{aligned}
$$

Here we used the fact that $r(t)^{2} \leq 2(n-1)\left(T_{0}-t\right)$. Choose $T_{*}<T_{0}$ closer to $T_{0}$ if necessary such that $T_{*}+4\left(T_{0}-T_{*}\right)<T_{+}$. Then if $t \geq T_{*}+4\left(T_{0}-T_{*}\right)$, one has $\tilde{r}(t)^{2} \leq 0$. This implies that $\tilde{T} \leq T_{*}+4\left(T_{0}-T_{*}\right)<T_{+}$.

\subsection{Initial data}

Given a target time $T$, we now construct initial data $u=1+v$ at $t=$ $t_{0}<T$ that will become singular at $T$ and satisfy the asymptotic profile (2.34). In the next subsection, we shall obtain appropriate estimates for the function $V(\sigma, \tau)=\eta\left(e^{-\delta \tau / 2} \sigma\right) \cdot v(\sigma, \tau)=\sum_{k=0}^{\infty} a_{2 k}(\tau) h_{2 k}(\sigma)$ corresponding to this construction.

Let $\varepsilon \in\left(0, \frac{1}{4 \sqrt{n-1}}\right)$ be a constant to be chosen later. Near the developing neckpinch, we impose the initial profile

$$
u=1+\alpha_{0} h_{0}(\sigma)+\alpha_{2} h_{2}(\sigma)
$$

where $\alpha_{0}$ and $\alpha_{2}>0$ are constants to be chosen later, and $\sigma(s, t)=s / \sqrt{T-t}$. That is, we prescribe $\psi\left(s, t_{0}\right)=R_{n}\left(t_{0}\right) \cdot u\left(\sigma, t_{0}\right)$ as

$$
\psi\left(s, t_{0}\right)=\sqrt{2(n-1)} \sqrt{T-t_{0}}\left[\left(1+\alpha_{0}-2 \alpha_{2}\right)+\alpha_{2} \frac{s^{2}}{T-t_{0}}\right]
$$


on the region $\mathcal{N}=\left\{s:|s| \leq s_{0}\right\}$, where $s_{0}$ is given by

$$
s_{0}=\varepsilon \sqrt{T-t_{0}} \log \frac{1}{T-t_{0}} .
$$

We will require

$$
-\varepsilon \leq \alpha_{0} \leq 2 \alpha_{2}
$$

and

$$
\frac{\varepsilon}{\tau_{0}} \leq \alpha_{2} \leq \frac{1}{\tau_{0}}
$$

where

$$
\tau_{0}=\log \frac{1}{T-t_{0}} \gg 1 .
$$

The radius of a neck of the form $(2.37)$ is

$$
r_{0}=\psi\left(0, t_{0}\right)=\sqrt{2(n-1)} \sqrt{T-t_{0}}\left(1+\alpha_{0}-2 \alpha_{2}\right) .
$$

Thus

$$
\psi=r_{0}+\sqrt{2(n-1)} \frac{\alpha_{2} s^{2}}{\sqrt{T-t_{0}}} .
$$

Since

$$
\psi_{s}=2 \sqrt{2(n-1)} \frac{\alpha_{2} s}{\sqrt{T-t_{0}}}
$$

we have $\left|\psi_{s}\right| \leq \psi_{s}\left(s_{0}, t_{0}\right)$ on $\mathcal{N}$, where $\psi_{s}\left(s_{0}, t_{0}\right)=2 \varepsilon \sqrt{2(n-1)} \cdot \tau_{0} \alpha_{2}$ satisfies

$$
\varepsilon^{2} \leq \frac{\psi_{s}\left(s_{0}, t_{0}\right)}{2 \sqrt{2(n-1)}} \leq \varepsilon .
$$

Outside $\mathcal{N}$, we extend $\psi$ linearly with slope $\psi_{s}\left(s_{0}, t_{0}\right)>0$ until we reach the height $\psi=1$, where we glue on a standard profile that is smooth at the poles. As in $[3, \S 8]$, this can be done with $\left|\psi_{s}\right| \leq 1$ everywhere. Notice that the initial profile $\psi\left(\cdot, t_{0}\right)$ is smooth except on $\partial \mathcal{N}$; by [31], the corresponding solution of Ricci flow will be smooth for all $t>t_{0}$ that it exists.

We next obtain a formula for the upper bound $F^{*}$ on the cylindricality $F$ of a neck of the form (2.37), where $\alpha_{0}$ and $\alpha_{2}$ satisfy (2.38) and (2.39), 
respectively. Note that $F \leq 0$ off $\mathcal{N}$. On $\mathcal{N}$, we have

$$
\begin{aligned}
\psi \leq \psi\left(s_{0}, t_{0}\right) & =r_{0}+\sqrt{2(n-1)} \frac{\alpha_{2} s_{0}^{2}}{\sqrt{T-t_{0}}} \\
& \leq \sqrt{2(n-1)}\left(1+\varepsilon^{2} \tau_{0}\right) \sqrt{T-t_{0}}
\end{aligned}
$$

and

$$
\psi_{s s}=2 \sqrt{2(n-1)} \frac{\alpha_{2}}{\sqrt{T-t_{0}}} \leq \frac{2 \sqrt{2(n-1)}}{\tau_{0} \sqrt{T-t_{0}}} .
$$

Independent of $\varepsilon \leq \frac{1}{4}$, we may choose $t_{*}$ close enough to $T$ so that for any $t_{0} \in\left[t_{*}, T\right)$, the radius is bounded from below by

$$
r_{0} \geq \sqrt{2(n-1)} \sqrt{T-t_{0}}\left(1-\varepsilon-\frac{2}{\tau_{0}}\right) \geq \frac{\sqrt{2(n-1)}}{2} \sqrt{T-t_{0}},
$$

and so that the sectional curvature $L$ is bounded from below on $\mathcal{N}$ by

$$
L=\frac{1-\psi_{s}^{2}}{\psi^{2}} \geq \frac{1-8(n-1) \varepsilon^{2}}{\psi\left(s_{0}, t_{0}\right)^{2}} \geq \frac{1}{4(n-1)\left(T-t_{0}\right)\left(1+\varepsilon^{2} \tau_{0}\right)^{2}} \geq e^{2}
$$

provided $\tau_{0}>\tau_{0}^{*}(n, \varepsilon)$. Here we used the fact that $\lim _{x} \searrow_{0}\left(x \log \frac{1}{x}\right)=0$. By $[3$, Lemma 9.1], the bound $L \geq e^{2}$ allows us to assume $\lambda=0$. Combining the estimates above, we conclude that there exists a $C=C\left(n, t_{*}\right)$ such that for any $t_{0} \in\left[t_{*}, T\right)$, one has

$$
\begin{aligned}
F=\frac{K}{L} \log L & =\frac{\psi \psi_{s s}}{1-\psi_{s}^{2}} \log \frac{1-\psi_{s}^{2}}{\psi^{2}} \\
& \leq \frac{2}{1-8(n-1) \varepsilon^{2}} \psi \psi_{s s} \log \frac{1}{r_{0}} \\
& \leq 16(n-1) \frac{1+\varepsilon^{2} \tau_{0}}{\tau_{0}}\left(\log 2+\frac{\tau_{0}}{2}\right) \\
& \leq C \varepsilon^{2} \tau_{0}
\end{aligned}
$$

Hence we may define

$$
F^{*}=(n-1)+C \varepsilon^{2} \tau_{0}
$$

This formula for $F^{*}$ allows us to adjust our constants in order to force pinching at $T$. 
Lemma 2.18. There exist an $\varepsilon_{*}$ and a $t_{*}$ depending only on $n$ such that for any $\varepsilon \in\left(0, \varepsilon_{*}\right]$, all initial times $t_{0} \in\left[t_{*}, T\right)$, and any $\alpha_{2}$ satisfying $(2.39)$, there exists some $\alpha_{0}$ satisfying (2.38) such that the solution with initial profile (2.37) becomes singular exactly at $T$.

Proof. Proposition 5.2 of [3] implies that $r$ is monotonically decreasing. So for all $t \geq t_{0}$ that the solution exists, one has

$$
r(t)^{2} \leq r_{0}^{2} \leq 2(n-1)\left(T-t_{0}\right)
$$

As above, we fix $t_{*}<T$ so that $(2.41)$ holds for all $t_{0} \in\left[t_{*}, T\right)$. Then by (2.36), there exists a $c>0$ depending only on $n$ such that

$$
-2(n-1) \leq \frac{d}{d t}\left(r^{2}\right) \leq-2(n-1)+2 \frac{n-1+C \varepsilon^{2} \tau_{0}}{\tau_{0}-c} .
$$

There exists a $C_{*}=C_{*}\left(n, t_{*}\right)$ such that $\left(n-1+C \varepsilon^{2} \tau_{0}\right) / \tau_{0}-c \leq C_{*} \varepsilon^{2}$. Hence we may fix $\varepsilon_{*}=\varepsilon_{*}\left(C_{*}\right)$ such that for all $\varepsilon \in\left(0, \varepsilon_{*}\right]$, one has

$$
-2(n-1) \leq \frac{d}{d t}\left(r^{2}\right) \leq-2(n-1)(1-\varepsilon) .
$$

By [3, Lemma 7.1], the solution becomes singular at $\tilde{T}$ if and only if $r(\tilde{T})=0$. It follows that

$$
\frac{r_{0}^{2}}{2(n-1)} \leq \tilde{T}-t_{0} \leq \frac{1}{1-\varepsilon} \cdot \frac{r_{0}^{2}}{2(n-1)} .
$$

By (2.40), this implies that

$$
\left(1+\alpha_{0}-2 \alpha_{2}\right)^{2} \leq \frac{\tilde{T}-t_{0}}{T-t_{0}} \leq \frac{\left(1+\alpha_{0}-2 \alpha_{2}\right)^{2}}{1-\varepsilon} .
$$

Thus if $\alpha_{0}-2 \alpha_{2}>0$, then pinching occurs at $\tilde{T}>T$. On the other hand, if $-1<\alpha_{0}-2 \alpha_{2}<-\varepsilon$, then pinching occurs at $\tilde{T}<T$. By Lemma 2.17, pinching will occur exactly at $T$ for some $\alpha_{0} \in\left[2 \alpha_{2}-\varepsilon, 2 \alpha_{2}\right]$. Clearly, this $\alpha_{0}$ satisfies $(2.38)$.

\subsection{The dominant asymptotic profile}

For $\tau \geq \tau_{0}$, let $v(\sigma, \tau)$ denote the solution of Ricci flow corresponding to the initial data (2.37). Then

$$
v\left(\sigma, \tau_{0}\right)=\alpha_{0} h_{0}(\sigma)+\alpha_{2} h_{2}(\sigma) \quad \text { for } \quad|\sigma| \leq \sigma_{0}=\sigma\left(s_{0}, t_{0}\right)=\varepsilon \tau_{0} .
$$


For $\tau \geq \tau_{0}$, define $V(\sigma, \tau)=\eta\left(e^{-\delta \tau / 2} \sigma\right) v(\sigma, \tau)$, noting that $V(\cdot, \tau) \in \mathcal{H}$ admits the orthogonal decomposition

$$
V(\sigma, \tau)=a_{0}(\tau) h_{0}(\sigma)+a_{2}(\tau) h_{2}(\sigma)+W(\sigma, \tau)
$$

Intuitively, one expects that $a_{0}$ and $a_{2}$ will be close to $\alpha_{0}$ and $\alpha_{2}$, respectively, at $\tau=\tau_{0}$ and for a short time thereafter. We now make this expectation precise. Define $U \in \mathcal{H}$ by

$$
U=V\left(\cdot, \tau_{0}\right)-\alpha_{0} h_{0}-\alpha_{2} h_{2}
$$

At $\tau=\tau_{0}$, one has $\left|V_{\sigma}\right|=\left|v_{\sigma}\right|=\left|\psi_{s}\right| / \sqrt{2(n-1)} \leq \varepsilon$ for $|\sigma| \leq \sigma_{0}$ and $\left|V_{\sigma}\right| \leq$ 1 everywhere. Because $\left|\alpha_{0}\right|, \alpha_{2} \in[0,1]$, there are universal constants $C$ (which may change from line to line) such that

$$
\begin{aligned}
U(\sigma)=0 & \text { for all }|\sigma|<\sigma_{0}, \\
|U(\sigma)| \leq C\left(1+\sigma^{2}\right) & \text { for all }|\sigma| \geq \sigma_{0}
\end{aligned}
$$

and hence such that

$$
\begin{aligned}
\|U\|^{2} & \leq C \int_{|\sigma| \geq \sigma_{0}}\left(1+\sigma^{2}\right)^{2} e^{-\sigma^{2} / 4} d \sigma \\
& \leq C e^{-\sigma_{0}^{2} / 5}=C e^{-\varepsilon^{2} \tau_{0}^{2} / 5} .
\end{aligned}
$$

Because $\|W\|^{2}=\langle W, W\rangle=\langle W, U\rangle \leq\|W\|\|U\|$ at $\tau=\tau_{0}$, it follows that the constant $W_{0}$ introduced in Lemma 2.5 satisfies

$$
W_{0}=e^{\tau_{0}}\left\|W\left(\cdot, \tau_{0}\right)\right\| \leq e^{\tau_{0}}\|U\| \leq C \exp \left(\tau_{0}-\frac{\varepsilon^{2}}{10} \tau_{0}^{2}\right)
$$

Similarly, one has

$$
a_{2}\left(\tau_{0}\right)=\frac{1}{\left\|h_{2}\right\|^{2}}\left\langle h_{2}, V\left(\cdot, \tau_{0}\right)\right\rangle=\alpha_{2}+\frac{1}{\left\|h_{2}\right\|^{2}}\left\langle h_{2}, U\right\rangle,
$$

and hence

$$
\left|a_{2}\left(\tau_{0}\right)-\alpha_{2}\right| \leq C e^{-\varepsilon^{2} \tau_{0}^{2} / 10}
$$

We are now ready to prove that alternative (2.34) occurs. By (2.32), one has

$$
\frac{d}{d \tau} a_{2}(\tau)=-8 a_{2}(\tau)^{2}+M^{*}(\tau)
$$


where $\left|M^{*}(\tau)\right| \leq C\left(1+W_{0}\right) \tau^{-3}$. By (2.43), we may make $\tau_{0}$ so large that $C\left(1+W_{0}\right) \leq C_{0}$. Then

$$
-8 a_{2}(\tau)^{2}-\frac{C_{0}}{\tau^{3}} \leq \frac{d}{d \tau} a_{2}(\tau) \leq-8 a_{2}(\tau)^{2}+\frac{C_{0}}{\tau^{3}} .
$$

By (2.39) and (2.44), we may make $\tau_{0}$ larger if necessary such that

$$
\sqrt{\frac{C_{0}}{8 \tau_{0}^{3}}} \ll \frac{\varepsilon}{2 \tau_{0}} \leq a_{2}\left(\tau_{0}\right) \leq \frac{2}{\tau_{0}} .
$$

As long as $a_{2}(\tau)^{2}>\frac{C_{0}}{8} \tau^{-3}$, the function $a_{2}(\tau)$ will be decreasing. For such times, one has

$$
\frac{d}{d \tau} a_{2}(\tau) \geq-8 a_{2}\left(\tau_{0}\right)^{2}-\frac{C_{0}}{\tau^{3}} \geq-\frac{32}{\tau_{0}^{2}}-\frac{C_{0}}{\tau_{0}^{3}} \geq-\frac{64}{\tau_{0}^{2}}
$$

provided that $\tau_{0}$ is large enough, and hence

$$
a_{2}(\tau) \geq a_{2}\left(\tau_{0}\right)-\frac{64}{\tau_{0}^{2}}\left(\tau-\tau_{0}\right) \geq \frac{\varepsilon}{2 \tau_{0}}-\frac{64}{\tau_{0}^{2}}\left(\tau-\tau_{0}\right) .
$$

In particular, given any $C>0$, one can make $\tau_{0}$ so large that

$$
a_{2}\left(\tau_{0}+1\right) \geq \frac{\varepsilon}{2 \tau_{0}}-\frac{64}{\tau_{0}^{2}} \geq C \frac{1+W_{0}}{\left(\tau_{0}+1\right)^{2}} .
$$

This justifies our assumption that $a_{2}$ was decreasing for $\tau_{0} \leq \tau \leq \tau_{0}+1$. Moreover, if we choose $C$ as in (2.33) then it violates alternative (2.33). Hence we have proved the following result.

Proposition 2.19. For any final time $T$, all sufficiently small $\varepsilon>0$, all initial times $t_{0}$ sufficiently close to $T$ and any $\alpha_{2}$ satisfying (2.39), there exists an $\alpha_{0}$ satisfying (2.38) such that the solution (2.42) with initial profile (2.37) becomes singular at $T$ and satisfies the asymptotic profile

$$
a_{2}(\tau)=\frac{1}{8 \tau}+\mathrm{o}\left(\frac{1}{\tau}\right) \quad \text { as } \tau \rightarrow \infty .
$$

\subsection{The intermediate region}

Consider a solution that forms a singularity at time $t=T$. Define $u, v$, etc. as above and assume that our solution satisfies (2.45). 
We have shown that

$$
v(\sigma, \tau)=\frac{\sigma^{2}-2}{8 \tau}+\mathrm{o}\left(\frac{1}{\tau}\right) \quad(\tau \rightarrow \infty),
$$

uniformly on any bounded interval $|\sigma| \leq A$.

Now consider the quantity

$$
q=u^{2}-1
$$

Lemma 2.20. For any constants $\varepsilon>0$ and $B<\infty$, there exist an $A$ and $a \bar{\tau}$ such that

$$
(1-\varepsilon) \frac{\sigma^{2}}{4 \tau}<q(\sigma, \tau)<(1+\varepsilon) \frac{\sigma^{2}}{4 \tau}
$$

whenever $A \leq \sigma \leq B \sqrt{\tau}$ and times $\tau \geq \bar{\tau}$.

In other words, for $1 \ll \sigma \leq B \sqrt{\tau}$ one has

$$
u=\sqrt{1+(1+\mathrm{o}(1)) \frac{\sigma^{2}}{4 \tau}} \approx \sqrt{1+\frac{\sigma^{2}}{4 \tau}} .
$$

We shall prove this lemma by computing the evolution equation for $q$ in the variables $\tau$ and $\rho=\sigma / \sqrt{ } \tau$, and finding suitable sub- and supersolutions. The evolution equation (2.49) turns out to be a small perturbation of a first order linear equation, which is easily solved by following its characteristics.

2.18.1. Equation for $q$. Since $q_{\sigma}=2 u u_{\sigma}$ and $q_{\sigma \sigma}=2 u u_{\sigma \sigma}+2 u_{\sigma}^{2}$, one has

$$
\left.q_{\tau}\right|_{x}=q_{\sigma \sigma}+q+\frac{n-2}{2} \frac{q_{\sigma}^{2}}{q+1} .
$$

Here the time derivative is with constant $x$. Using Lemma 2.2, we get

$$
\left.q_{\tau}\right|_{\sigma}=q_{\sigma \sigma}-\frac{\sigma}{2} q_{\sigma}+q-n J(\sigma, \tau) q_{\sigma}+\frac{n-2}{2} \frac{q_{\sigma}^{2}}{q+1},
$$

where $\left.q_{\tau}\right|_{\sigma}$ is the derivative of $q$ with $\sigma$ held constant and where

$$
J=\frac{u_{\sigma}}{u}+\int_{0}^{\sigma} \frac{u_{\sigma}^{2}}{u^{2}} d \sigma .
$$

We now regard $q$ as a function of the new variable $\rho=\sigma / \sqrt{\tau}$. Because

$$
\left.q_{\tau}\right|_{\sigma}=\left.q_{\tau}\right|_{\rho}-\frac{1}{2} \frac{\rho}{\tau} q_{\rho}
$$


one has

$$
\left.q_{\tau}\right|_{\rho}-\frac{1}{\tau} q_{\rho \rho}+\frac{\rho}{2} q_{\rho}-q=\frac{1}{\tau}\left\{\frac{\rho}{2} q_{\rho}-n \sqrt{\tau} J q_{\rho}+\frac{n-2}{2} \frac{q_{\rho}^{2}}{q+1}\right\}
$$

2.18.2. A subsolution for (2.49). We begin with some simple estimates. Fix some $A_{0} \gg 1$ and recall that $\tau_{0}$ is the smallest time under consideration in this paper. Given $B>1$, we shall choose $A \geq A_{0}$ and $\tau_{1} \geq \tau_{0}$ large enough to make everything work. The region $A / \sqrt{\tau} \leq \rho \leq B^{2}$ corresponds to $A \leq$ $\sigma \leq B^{2} \sqrt{\tau}$. By Lemma 2.1, there exist $C_{0}=C_{0}(B)$ and $C_{1}=C_{1}\left(A_{0}\right)$ such that for all $A \leq \sigma \leq B^{2} \sqrt{\tau}$, one has

$$
u \leq 1+C_{0} \frac{\sigma^{2}}{\tau}=1+C_{0} \rho^{2} \leq 1+C_{0} B^{4}
$$

and

$$
0 \leq u_{\sigma} \leq C_{0} \frac{1+\sigma}{\tau} \leq C_{1} \frac{\sigma}{\tau}=C_{1} \frac{\rho}{\sqrt{\tau}}
$$

Thus we have

$$
0 \leq u_{\rho} \leq C_{1} \rho
$$

and

$$
0 \leq \rho q_{\rho}=2 \rho u_{\rho} u \leq C\left(A_{0}, B\right)
$$

for $A / \sqrt{\tau} \leq \rho \leq B^{2}$. Since $u \geq 1-C / \tau$ for $C=C\left(n, F^{*}\right)$, we may assume without loss of generality that $\tau_{0} \gg 1$ is so large that

$$
\frac{q_{\rho}^{2}}{q+1}=\frac{q_{\rho}^{2}}{u^{2}} \leq 2 q_{\rho}^{2} \leq C\left(A_{0}, B\right)
$$

for $A / \sqrt{\tau} \leq \rho \leq B^{2}$ and $\tau \geq \tau_{0}$. Since $n \sqrt{\tau} J q_{\rho} \geq 0$ for all $0 \leq \sigma \leq e^{\delta \tau}$, it follows that

$$
\frac{\rho}{2} q_{\rho}-n \sqrt{\tau} J q_{\rho}+\frac{n-2}{2} \frac{q_{\rho}^{2}}{q+1} \leq C\left(A_{0}, B\right)
$$

We introduce the linear differential operator

$$
\left.\mathcal{L}[f] \doteqdot f_{\tau}\right|_{\rho}-\frac{1}{\tau} f_{\rho \rho}+\frac{\rho}{2} f_{\rho}-f .
$$


Then (2.49) and (2.50) imply that $q=u^{2}-1$ satisfies

$$
|\mathcal{L}[q]| \leq \frac{C\left(A_{0}, B\right)}{\tau}
$$

whenever $A / \sqrt{\tau} \leq \rho \leq B^{2}$ and $\tau \geq \tau_{0}$.

Let $Q$ be a smooth function with support in [0,1] such that $Q(r) \leq r^{2} / 4$, and define

$$
K_{0}=\max _{0 \leq r \leq 1}\left|Q^{\prime \prime}(r)\right|
$$

For $\tau_{1} \geq \tau_{0}$, define

$$
q_{0}(\rho, \tau)=\rho_{1}(\tau)^{2} Q\left(\rho / \rho_{1}(\tau)\right)
$$

with

$$
\rho_{1}(\tau) \doteqdot e^{\left(\tau-\tau_{1}\right) / 2} \frac{A}{\sqrt{\tau}_{1}} .
$$

This function is almost in the nullspace of the linear operator $\mathcal{L}$; indeed, a straightforward computation shows that

$$
\mathcal{L}\left[q_{0}\right]=-\frac{1}{\tau} \frac{\partial^{2} q_{0}}{\partial \rho^{2}}=-\frac{1}{\tau} Q^{\prime \prime}\left(\rho / \rho_{1}(\tau)\right),
$$

so that $\left|\mathcal{L}\left[q_{0}\right]\right| \leq K_{0} / \tau$.

Now for $K \geq K_{0}$ to be determined and any $\theta \in(1 / 2,1)$, consider

$$
q_{-}(\rho, \tau)=\theta q_{0}(\rho, \tau)+\frac{K}{\tau}
$$

One then has

$$
\mathcal{L}\left[q_{-}\right]=\theta \mathcal{L}\left[q_{0}\right]+K \mathcal{L}\left[\tau^{-1}\right]<\theta \frac{K_{0}}{\tau}-\frac{K}{\tau}<\frac{K_{0}-K}{\tau} \leq 0
$$

so that $q_{-}$is a subsolution for all $\tau \geq \tau_{0}$. Moreover, if we choose $K \geq K_{0}+$ $C\left(A_{0}, B\right)$, then

$$
\mathcal{L}\left[q_{-}-q\right] \leq \frac{C\left(A_{0}, B\right)+K_{0}-K}{\tau} \leq 0
$$

for $A / \sqrt{\tau} \leq \rho \leq B^{2}$ and $\tau \geq \tau_{0}$. 
Define the region

$$
\Omega=\left\{(\rho, \tau): \frac{A}{\sqrt{\tau}} \leq \rho \leq \rho_{1}(\tau), \tau_{1} \leq \tau \leq \omega\right\}
$$

where $\omega$ is determined by $\rho_{1}(\omega)=B^{2}$, i.e.,

$$
\omega=\tau_{1}+\log \left(B^{4} \tau_{1} / A^{2}\right) .
$$

We will choose $A$ and $\tau_{1}$ so that $B^{4} \tau_{1}>A^{2}$. First, we compare the values of $q$ and $q_{-}$along the parabolic boundary of $\Omega$.

Along the left edge $\rho=A / \sqrt{\tau}, \tau \geq \tau_{1}$, it follows from (2.46) that

$$
q(A / \sqrt{\tau}, \tau)=u^{2}(A, \tau)-1=\frac{A^{2}-2}{4 \tau}+\mathrm{o}\left(\frac{1}{\tau}\right) \geq\left(1-\varepsilon_{0}\right) \frac{A^{2}}{4 \tau}
$$

where $\varepsilon_{0}=\varepsilon_{0}\left(A_{0}, \tau_{1}\right)$. On the other hand, there exists an $\varepsilon_{1}=\varepsilon_{1}\left(A, K, \tau_{0}\right)$ such that

$$
q_{-}(\rho, \tau) \leq \frac{\theta}{4} \rho^{2}+\frac{K}{\tau} \leq\left(1+\varepsilon_{1}\right) \frac{\theta}{4} \rho^{2}
$$

for $\rho \geq A / \sqrt{\tau}$. So $q_{-}<q$ at $(A / \sqrt{\tau}, \tau)$ provided that

$$
\frac{1}{2} \leq \theta<\frac{1-\varepsilon_{0}}{1+\varepsilon_{1}}
$$

Along the right edge $\rho=\rho_{1}(\tau)$, one has

$$
q_{-}\left(\rho_{1}(\tau), \tau\right)=\rho_{1}(\tau)^{2} Q(1)+\frac{K}{\tau}=\frac{K}{\tau},
$$

because $Q$ is supported in $[0,1]$. But since $q_{\rho} \geq 0$, estimate (2.55) implies that

$$
q\left(\rho_{1}(\tau), \tau\right)=q\left(e^{\left(\tau-\tau_{1}\right) / 2} A / \sqrt{\tau_{1}}, \tau\right) \geq q(A / \sqrt{\tau}, \tau) \geq \frac{1-\varepsilon_{0}}{4} \frac{A^{2}}{\tau}
$$

where $\varepsilon_{0}=\varepsilon_{0}\left(A_{0}, \tau_{1}\right)$. So $q_{-} \leq q$ at $\left(\rho_{1}(\tau), \tau\right)$ provided that

$$
A \geq 2 \sqrt{\frac{K}{1-\varepsilon_{0}}} .
$$

The bottom of $\Omega$ is the single point $\left(A / \sqrt{\tau_{1}}, \tau_{1}\right)$. So the choice $\theta<(1-$ $\left.\varepsilon_{0}\right) /\left(1+\varepsilon_{1}\right)$ ensures that $q_{-}<q$ there as well. 
Now choose $K$ depending on $A_{0}, B$ and $K_{0}$ to satisfy $K \geq K_{0}+C\left(A_{0}, B\right)$. Take $\tau_{1} \geq \tau_{0}$ large enough so that $\varepsilon_{0}<1 / 2$ and choose $A \geq A_{0}$ depending on $K$ so that $A \geq \sqrt{8 K}$ and so that $\varepsilon_{1}>0$ is as small as desired. Then increase $\tau_{1}$ if necessary so that $\tau_{1} \geq A^{2} / B^{4}$, making $\varepsilon_{0}$ as small as desired. These choices make it possible to take $\theta \in(1 / 2,1)$ as close to 1 as one wishes and still satisfy $\theta<\left(1-\varepsilon_{0}\right) /\left(1+\varepsilon_{1}\right)$. Furthermore, they ensure that $q_{-} \leq q$ along the parabolic boundary of $\Omega$. Because $e^{\left(\tau-\tau_{1}\right) / 2} A / \sqrt{\tau_{1}} \leq B^{2}$ for all $\tau \leq \omega$, it follows from (2.53) that $q \geq q_{-}$throughout $\Omega$. Since for any $\varepsilon$, one may take $Q(r)=r^{2} / 4$ for $r \in(\varepsilon, 1-\varepsilon)$, this implies in particular that

$$
q(\rho, \tau)>\theta \frac{\rho^{2}}{4}
$$

for all points $\rho$ such that $A / \sqrt{\tau} \leq \rho \leq B$ and times $\tau$ such that

$$
2 \log \left(B \sqrt{\tau_{1}} / A\right) \leq \tau-\tau_{1} \leq 2\left[\log \left(B \sqrt{\tau_{1}} / A\right)+\log B\right]
$$

Our estimates only improve if we increase $\tau_{1}$ (without altering any other choices); in particular, $\theta$ does not decrease. Since $\tau=\tau_{1}+\mathrm{o}\left(\tau_{1}\right)$ as $\tau_{1} \rightarrow \infty$, we have proved the lower estimate in Lemma 2.20.

2.18.3. A supersolution. A slight modification of the preceding construction will give an upper barrier for $q$ on the same domain $\Omega$. One begins with the a priori bound

$$
u \leq C\left(1+\sigma^{2} / \tau\right)=C\left(1+\rho^{2}\right) \text { and hence } q \leq C\left(1+\rho^{2}\right) \rho^{2}=C_{0}(B) \rho^{2},
$$

which holds for $A_{0} / \sqrt{\tau} \leq \rho \leq B^{2}$.

Choose a smooth function $\hat{Q}$ which satisfies $\hat{Q}(r) \geq r^{2} / 4$, and define $\hat{K}_{0}=\max _{[0,1]}\left|\hat{Q}^{\prime \prime}\right|$. Instead of requiring $\hat{Q}$ to be supported in $[0,1]$, we impose

$$
\hat{Q}(1)=2 C_{0}(B) \text { with } C_{0}(B) \text { as in }(2.56) .
$$

For $\tau \geq \tau_{1}$, one introduces $\hat{q}_{0}(\rho, \tau)=\rho_{1}(\tau)^{2} \hat{Q}\left(\rho / \rho_{1}(\tau)\right)$, as in (2.51), and finds that $\left|\mathcal{L}\left[\hat{q}_{0}\right]\right| \leq \hat{K}_{0} / \tau$. For $\hat{K} \geq \hat{K}_{0}$ and any $\theta \in\left(1, \frac{3}{2}\right)$, define

$$
q_{+}(\rho, \tau)=\theta \hat{q}_{0}(\rho, \tau)-\hat{K} / \tau .
$$

Choosing $\hat{K} \geq \hat{K}_{0}+C\left(A_{0}, B\right)$, one finds, as in (2.53), that $\mathcal{L}\left[q_{+}-q\right] \geq 0$ for $\tau \geq \tau_{0}$ and $A / \sqrt{\tau} \leq \rho \leq B^{2}$. Thus $q_{+}$is a supersolution, provided $q_{+} \geq q$ on the parabolic boundary of $\Omega$. 
On the left edge of $\Omega$, one has, as in $(2.55), q \leq\left(1+\varepsilon_{0}\right) A^{2} / 4 \tau$. One also has $q_{+} \geq\left(1-\varepsilon_{1}\right) \theta A^{2} / 4 \tau$, where $\varepsilon_{1}=\varepsilon_{1}(A, K)$.

Hence one will have $q \leq q_{+}$on the left edge if $\theta$ satisfies

$$
\frac{1+\varepsilon_{0}}{1-\varepsilon_{1}}<\theta<\frac{3}{2}
$$

On the right edge of $\Omega$ one has $\rho=\rho_{1}(\tau)$, and thus

$$
q_{+}(\rho, \tau)=\theta \rho_{1}(\tau)^{2} \hat{Q}(1)-\hat{K} / \tau \geq \theta\left(1-\hat{K} / A^{2}\right) Q(1) \rho_{1}(\tau)^{2} Q(1) \rho_{1}(\tau)^{2},
$$

provided $A$ is chosen so large that $\theta\left(1-\hat{K} / A^{2}\right)>1$. On the other hand we know that $q \leq C_{0}(B) \rho_{1}(\tau)^{2}$ holds on the right edge of $\Omega$. Hence our condition $\hat{Q}(1)>C_{0}(B)$ implies that $q_{+}>q$ on the right edge.

It now follows that $q \leq q_{+}$on $\Omega$, and as with the subsolution, this implies that $q(\rho, \tau)<\theta \rho^{2} / 4$ for all $\rho \in[A / \sqrt{\tau}, B]$ and $\tau \geq \tau_{1}+\mathrm{o}\left(\tau_{1}\right)$.

\subsection{Convergence in the outer region}

Let $B$ and $\varepsilon>0$ be given constants. Define $A=A(\varepsilon, B)$ and $\bar{\tau}=\bar{\tau}(\varepsilon, B)$ as in Lemma 2.20. Then we have shown that when

$$
\sigma=B \sqrt{\tau}, \quad \text { i.e., when } s^{2}=\frac{B^{2}}{4}(T-t) \log \frac{1}{T-t},
$$

one has

$$
\sqrt{1+(1-\varepsilon) B^{2} / 4} \leq u \leq \sqrt{1+(1+\varepsilon) B^{2} / 4}
$$

For $\psi=R_{n}(t) u$, this therefore means that

$$
\sqrt{T-t} \sqrt{1+(1-\varepsilon) B^{2} / 4} \leq \frac{\psi}{\sqrt{2(n-1)}} \leq \sqrt{T-t} \sqrt{1+(1+\varepsilon) B^{2} / 4}
$$

for all $t \in[\bar{t}, T)$, where $\bar{t}=T-e^{-\bar{\tau}}$.

We define the outer region (with parameter $B$ ) to be the portion of space-time given by

$$
\Gamma_{B}=\left\{(x, t): \bar{\tau} \leq \tau<T, s(x, t)^{2} \geq \frac{B^{2}}{4}(T-t) \log \frac{1}{T-t}\right\} .
$$

The boundary $\partial \Gamma_{B}$ of $\Gamma_{B}$ is given by (2.57). 
For any given $t_{*} \in[\bar{t}, T)$, we let $x_{*}$ be the $x$ coordinate of the point on $\partial \Gamma_{B}$ with $t=t_{*}$. We will now show that $s\left(x_{*}, t\right)$ and $\psi\left(x_{*}, t\right)$ change very little for $t_{*} \leq t<T$. This will directly imply the asymptotic description of $\psi(s, T)$ for $s \rightarrow 0$ in the introduction. Fix some $B_{0}>1$. We begin with a simple uniform estimate for $\psi_{s}$ in the region $B_{0} \sqrt{\tau} \leq \sigma \leq \eta e^{\delta \tau}$.

Lemma 2.21. For any $B_{0}>1$, there exist constants $\gamma, \delta>0$ and $C<\infty$ depending only on $B_{0}$ and the initial data such that

$$
0 \leq \psi_{s}(s, t) \leq \frac{C}{\sqrt{\tau}}
$$

for all $\tau \geq \bar{\tau}\left(B_{0}\right)$ and $B_{0} \sqrt{\tau} \leq \sigma(s) \leq \gamma e^{\delta \tau}$

Proof. Define $r(t)=\psi(0, t)$. By (2.45), we have

$$
r=\sqrt{2(n-1)(T-t)}\left\{1-\frac{1}{4 \tau}+\mathrm{o}\left(\frac{1}{\tau}\right)\right\} .
$$

By (2.58), we have

$$
\psi(s, t) \geq \psi(\bar{s}, t) \geq \sqrt{2(n-1)(T-t)} \sqrt{1-B_{0}^{2} / 8}
$$

for all $s \geq \bar{s}\left(B_{0}\right)=B_{0} \tau \sqrt{T-t}$ and $\tau \geq \bar{\tau}\left(B_{0}\right) \geq \tau_{0}$. Now restricting our attention to the region of the neck where $\psi \leq 1 / B_{0}$ and $0 \leq \psi_{s} \leq 1 / B_{0}$, we may apply estimate (34) of [3], obtaining $C$ depending on $B_{0}$ and $F^{*}$ such that

$$
\psi_{s}^{2} \leq C \log \left(\frac{\log r}{\log \psi}\right)
$$

This estimate holds as long as $\sigma \leq \gamma e^{\delta \tau}$, i.e., as long as $s \leq \gamma(\sqrt{T-t})^{1-2 \delta} \leq$ $r^{1-2 \delta}$. Let $c_{1}=\log \sqrt{1-B_{0}^{2} / 8}$. Then

$$
\frac{\log r}{\log \psi} \leq \frac{\log \sqrt{2(n-1)}-\tau / 2-1 / 4 \tau+\mathrm{o}(1 / \tau)}{\log \sqrt{2(n-1)}-\tau / 2+c_{1}}=1+\frac{2 c_{1}}{\tau}+\mathrm{o}\left(\frac{1}{\tau}\right)
$$

Hence $\tau \psi_{s}^{2} \leq C \tau \log (\log r / \log \psi)=C\left(2 c_{1}+\mathrm{o}(1)\right)$.

We are now ready to describe $s\left(x_{*}, t\right)$ and $\psi\left(x_{*}, t\right)$. 
Lemma 2.22. For any $x_{*}$ the limits

$$
\lim _{t \rightarrow T} s\left(x_{*}, t\right) \quad \text { and } \quad \lim _{t \rightarrow T} \psi\left(x_{*}, t\right)
$$

exist. Furthermore, a constant $C<\infty$, which does not depend on $B \geq B_{0}$ or $x_{*}$, exists for which one has

$$
\left|s\left(x_{*}, t\right)-s\left(x_{*}, t_{*}\right)\right| \leq C\left\{B^{-3}+\left|\log \left(T-t_{*}\right)\right|^{-3 / 2}\right\} s\left(x_{*}, t_{*}\right)
$$

and

$$
\left|\psi\left(x_{*}, t\right)-\psi\left(x_{*}, t_{*}\right)\right| \leq \frac{C}{B^{2}} \psi\left(x_{*}, t_{*}\right)
$$

for all $t \in\left[t_{*}, T\right)$.

Proof. To estimate the change in $s\left(x_{*}, t\right)$ we use

$$
\left.\frac{\partial s}{\partial t}\right|_{x=x_{*}}=n \frac{\psi_{s}\left(x_{*}, t\right)}{\psi\left(x_{*}, t\right)}+n \int_{0}^{s\left(x_{*}, t\right)}\left(\frac{\psi_{s}}{\psi}\right)^{2} d s,
$$

as in the proof of Lemma 1.2. Let $t^{\prime} \in\left(t_{*}, T\right)$ be given, and let $x^{\prime}$ be such that $s\left(x^{\prime}, t^{\prime}\right)$ lies on $\partial \Gamma_{B}$.

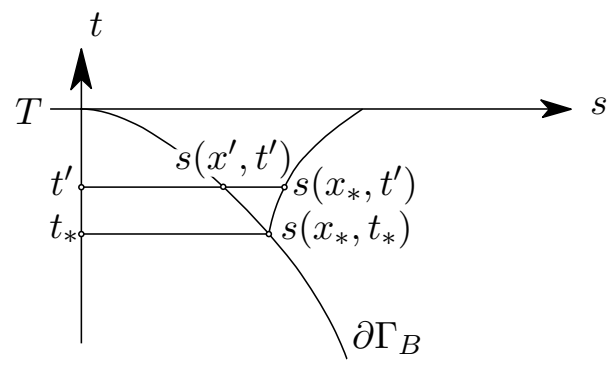

Then at time $t^{\prime}$, Lemma 2.21 implies that

$$
0 \leq \psi_{s}\left(s, t^{\prime}\right) \leq \frac{C}{\sqrt{\log 1 /\left(T-t^{\prime}\right)}} \quad \text { for all } s \leq s^{\prime}
$$

Since $x_{*} \geq x^{\prime}$, we then get

$$
\int_{0}^{s\left(x_{*}, t^{\prime}\right)}\left(\frac{\psi_{s}}{\psi}\right)^{2} d s=\int_{0}^{s\left(x^{\prime}, t^{\prime}\right)}\left(\frac{\psi_{s}}{\psi}\right)^{2} d s+\int_{s\left(x^{\prime}, t^{\prime}\right)}^{s\left(x_{*}, t^{\prime}\right)}\left(\frac{\psi_{s}}{\psi}\right)^{2} d s .
$$


In the first term, we use (2.62) to get

$$
\begin{aligned}
\int_{0}^{s\left(x^{\prime}, t^{\prime}\right)}\left(\frac{\psi_{s}}{\psi}\right)^{2} d s & \leq \frac{C}{\sqrt{\ln 1 /\left(T-t_{*}\right)}} \int_{0}^{s\left(x^{\prime}, t^{\prime}\right)} \frac{\psi_{s}}{\psi^{2}} d s \\
& \leq \frac{C}{\sqrt{\ln 1 /\left(T-t_{*}\right)}} \frac{1}{\psi\left(0, t^{\prime}\right)} \\
& \leq \frac{C}{\sqrt{\left(T-t^{\prime}\right) \ln 1 /\left(T-t_{*}\right)}}
\end{aligned}
$$

In the second term, we have $x^{\prime}<x<x_{*}$ such that

$$
\psi \geq \psi\left(x^{\prime}, t^{\prime}\right) \geq \sqrt{2(n-1)\left(T-t^{\prime}\right)\left(1+(1-\varepsilon) B^{2} / 4\right)} \geq C B \sqrt{T-t^{\prime}}
$$

with $C$ independent of $t$ or $B \geq B_{0}$. On the other hand, we also have $\psi_{s} \leq 1$ such that

$$
\begin{aligned}
\int_{s\left(x^{\prime}, t^{\prime}\right)}^{s\left(x_{*}, t^{\prime}\right)}\left(\frac{\psi_{s}}{\psi}\right)^{2} d s & \leq \int_{s\left(x^{\prime}, t^{\prime}\right)}^{s\left(x_{*}, t^{\prime}\right)}\left(\frac{\psi_{s}}{\psi^{2}}\right) d s \\
& \leq \frac{1}{\psi\left(x^{\prime}, t^{\prime}\right)} \\
& \leq \frac{C}{B \sqrt{T-t^{\prime}}}
\end{aligned}
$$

For the first term in (2.61), one has the same estimate, so that at any time $t^{\prime} \in\left(t_{*}, T\right]$, one has

$$
0 \leq \partial_{t} s\left(x_{*}, t^{\prime}\right) \leq C\left\{B^{-1}+\left|\log \left(T-t_{*}\right)\right|^{-1 / 2}\right\}\left(T-t^{\prime}\right)^{-1 / 2} .
$$

This is integrable in $t^{\prime} \in\left(t_{*}, T\right)$, so that $\lim _{t \rightarrow T} s\left(x_{*}, t\right)$ must exists. Integration with $t_{*}<t^{\prime}<t$ then leads to

$$
\left|s\left(x_{*}, t\right)-s\left(x_{*}, t_{*}\right)\right| \leq C\left\{B^{-1}+\left|\log \left(T-t_{*}\right)\right|^{-1 / 2}\right\} \sqrt{T-t_{*}} .
$$

Recall (2.57), apply $a^{2} b, a b^{2} \leq a^{3}+b^{3}$ to $a=B^{-1}$ and $b=\left|\log \left(T-t_{*}\right)\right|^{-1 / 2}$, and conclude

$$
\begin{aligned}
\left|s\left(x_{*}, t\right)-s\left(x_{*}, t_{*}\right)\right| & \leq C\left\{B^{-1}+\left|\log \left(T-t_{*}\right)\right|^{-1 / 2}\right\} \frac{s\left(x_{*}, t_{*}\right)}{B \sqrt{\ln 1 /\left(T-t_{*}\right)}} \\
& \leq C\left\{B^{-3}+\left|\log \left(T-t_{*}\right)\right|^{-3 / 2}\right\} s\left(x_{*}, t_{*}\right),
\end{aligned}
$$

which proves the estimate (2.59) for $s$. 
To establish the other estimate, we recall from [3, Proposition 5.3] that there is a constant $C$ which only depends on the initial data and the dimension $n$ such that

$$
\left|2 \psi \psi_{t}\right| \leq C
$$

always holds. In particular, the constant $C$ does not depend on our choice of $B$. In [3], we used this estimate to show that $\lim _{t \rightarrow T} \psi\left(x_{*}, t\right)$ exists. It also implies that

$$
\left|\psi\left(x_{*}, t\right)^{2}-\psi\left(x_{*}, t_{*}\right)^{2}\right| \leq C\left(t-t_{*}\right)
$$

and hence, using (2.58), that

$$
\left|\psi\left(x_{*}, t\right)-\psi\left(x_{*}, t_{*}\right)\right| \leq \frac{C\left(t-t_{*}\right)}{\psi\left(x_{*}, t\right)+\psi\left(x_{*}, t_{*}\right)} \leq \frac{C}{B} \sqrt{T-t_{*}} \leq \frac{C}{B^{2}} \psi\left(x_{*}, t_{*}\right)
$$

for all $t \in\left(t_{*}, T\right)$.

\subsection{Asymptotics in the outer region}

Using (2.58) and (2.60), we compute that

$$
\begin{aligned}
\psi\left(x_{*}, t\right) & =\left(1+\mathcal{O}\left(B^{-2}\right)\right) \psi\left(x_{*}, t_{*}\right) \\
& =\left(1+\mathcal{O}\left(B^{-2}\right)\right) \sqrt{2(n-1)\left[1+(1+\theta \varepsilon) B^{2} / 4\right]\left(T-t_{*}\right)} \\
& =\left(1+\mathcal{O}\left(B^{-2}+\varepsilon\right)\right) \sqrt{\frac{n-1}{2}} B \sqrt{T-t_{*}}
\end{aligned}
$$

for some $|\theta| \leq 1$.

¿From the definition (2.57), we see that $s=s\left(x_{*}, t_{*}\right)$ satisfies

$$
B \sqrt{T-t}=\frac{s}{\sqrt{-\log (T-t)}} .
$$

Hence, taking logarithms and abbreviating $\omega=T-t$,

$$
\log B+\frac{1}{2} \ln \omega=\log s-\frac{1}{2} \ln |\log \omega|,
$$

so that

$$
\begin{aligned}
\log \omega & =2 \ln s-\log |\log \omega|-2 \ln B=\mathcal{O}(\log s) \\
& =2 \ln s+\mathcal{O}(\log |\log s|) \\
& =(2+\mathrm{o}(1)) \log s
\end{aligned}
$$


We therefore find that

$$
B \sqrt{\omega}=(1+\mathrm{o}(1)) \frac{s}{\sqrt{-2 \ln s}} .
$$

Thus as $t_{*} \rightarrow T$, one has

$$
\psi=\left(1+\mathcal{O}\left(B^{-2}+\varepsilon\right)\right)\left(\frac{1}{2} \sqrt{n-1}+o(1)\right) \frac{s}{\sqrt{-\log s}}
$$

for all $t \in\left[t_{*}, T\right]$ and $x_{*}$ for which $s=s\left(x_{*}, t_{*}\right)$ satisfies (2.57). Since $B$ and $\varepsilon$ can be chosen arbitrarily, this implies

Lemma 2.23. For any $\delta>0$ there exist $a B>0$ and $a \bar{t}<T$ such that

$$
\left(\frac{1}{2} \sqrt{n-1}-\delta\right) \frac{s}{\sqrt{-\log s}} \leq \psi \leq\left(\frac{1}{2} \sqrt{n-1}+\delta\right) \frac{s}{\sqrt{-\log s}}
$$

in the region $(T-t)|\log (T-t)| \leq s^{2} / B^{2}, \bar{t} \leq t \leq T$.

\section{Formal matched asymptotics for general neckpinches}

The usual starting point for obtaining matched asymptotic expansions near a stationary solution of a nonlinear PDE is to linearize around that solution. This method encounters two obstacles when applied to Ricci flow, both of which can already be seen in our rigorous treatment of the symmetric neckpinch in Section 2.

The first challenge is the well-known fact that Ricci flow is only weakly parabolic. This deficiency stems from its invariance under the full diffeomorphism group of the underlying manifold.

In Section 2, we overcame this problem by using the geometrically defined quantity $s$ as a new coordinate, which led us to the parabolic PDE (2.16). For the general neckpinch without symmetry, we could find no simple ana$\log$ of the $s$ variable; and so, instead, we shall use a variant of the DeTurck trick $[11,12]$. This motivates our choice of $Y$ in Section 3.4 below, a choice which is equivalent to the Bianchi gauge, as adopted in the elliptic context by Biquard [7] and others. Note that solutions of DeTurck flow are equivalent to solutions of Ricci flow, modulo diffeomorphisms.

The second challenge is the fact that the linearized PDE possesses a null eigenvalue, suggesting the presence of a center manifold. This phenomenon also occurs in neckpinches of the mean curvature flow [5], and in 


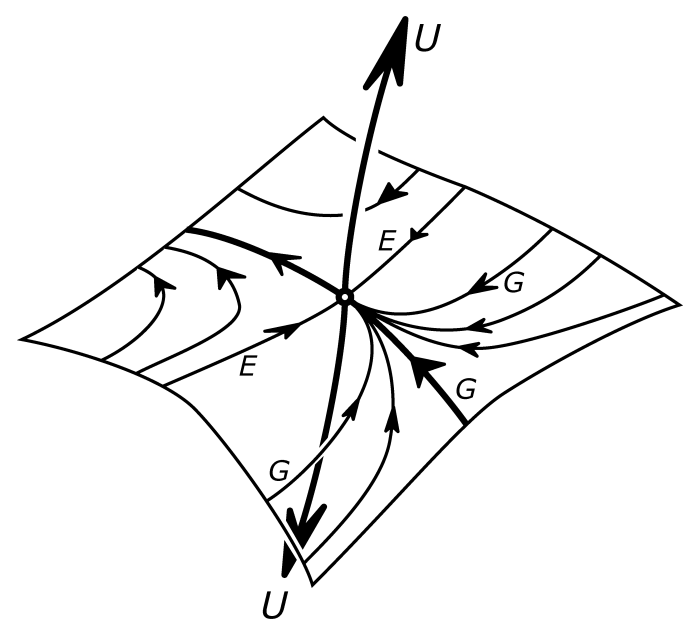

Figure 1: Renormalized Ricci flow near the cylinder.

generic singular solutions to the reaction diffusion equation $\partial_{t} u=\Delta u+u^{p}$ (see $[15,22,23])$. The null eigenvalue appears as follows. After introducing a renormalized Ricci flow for which the cylinder is a stationary solution, we find that the deviation $h \doteqdot g-g_{\text {cyl }}$ from the straight cylinder satisfies a nearly linear equation of the form

$$
\partial_{\tau} h=A h+N(h),
$$

where $A$ is a symmetric linear operator and $N(h)$ contains the higher-order nonlinear terms (cf. (2.16) for the symmetric case in which we had $v$ instead of $h$ ). Here $\tau=-\log (T-t)$, and $T$ is the blow-up time.

The operator $A$ is diagonalizable. It has a finite number of positive eigenvalues, an unbounded sequence of negative eigenvalues, and it also has zero as an eigenvalue. To guess what solutions near the cylinder, i.e., small solutions of (3.1), will do, we imagine for a moment that (3.1) is a system of ODE. In that situation one would use the decomposition into eigenfunctions of $A$ to split $h$ into stable, unstable and neutral components, corresponding to positive, negative and zero eigenvalues, respectively. The linear part of the Equation (3.1) will cause the unstable part to grow exponentially and the stable part to decay exponentially. The evolution of the neutral part is determined by the nonlinear terms. (See figure 1 for a caricature.)

Most solutions may get close to the stationary state $h=0$ but will eventually veer off along the exponentially growing unstable direction ("U" in 
figure 1). These solutions of (3.1) correspond to solutions of Ricci flow which do not form a neck pinch at the time $T$ or at the location on $S^{n+1}$ about which we renormalized the flow. However, for suitable initial data, the unstable component of $h$ will never grow. For such solutions one expects the neutral components to be dominant. If they decay to zero, then they will do so more slowly than exponentially ( $\sim \tau^{-1}$ in our case). The stable and unstable components will then also decay at a less than exponential rate, due to the nonlinear coupling in (3.1). Such solutions are marked "G" in figure 1, and these are the solutions which we attempt to describe with the formal computations below. There is a third possibility, namely, that for certain exceptional choices of initial data, the unstable components are always the largest. In this case, $h$ will decay exponentially. Such solutions are marked "E" in figure 1. In the case of the neck pinch for mean curvature flow, such exceptional solutions were rigorously constructed in [5].

To compute the "generic singular solutions $G$ " we assume the solution is of the form $\bar{h}+\Phi(\bar{h})$, where $\bar{h}$ belongs to the kernel of the linearized operator $A$, and $\Phi(\bar{h}) \perp \bar{h}$ is at least quadratic in its dependence on $\bar{h}$. (See Section 3.9.) To compute the formal dynamics, we substitute this Ansatz into the dilated Ricci flow, project onto the kernel of the linearization, and compute the purely quadratic terms, yielding an ODE on the kernel. The errors introduced by this method should be smaller than the order of the solution, namely $\mathcal{O}\left(\tau^{-2}\right)$.

In summary, we assume in what follows that a singularity forms at a given place and time. We then construct approximate solutions which become singular at the given space-time point and exhibit a (presumably generic) $\tau^{-1}$ rate of singularity formation. We derive matched asymptotics for these approximate solutions. These agree with our rigorous results for the rotationally symmetric solutions studied in Section 2 above. A rigorous justification of the matched asymptotics derived below without symmetry assumptions would require one to show, e.g., by adapting the methods of [5], that suitably small perturbations of our approximate solutions become bona fide solutions.

We now proceed to pursue the method summarized above. The computations required are extensive, but the conclusion obtained is eminently simple. The formal matched asymptotics we derive here tell us that the asymptotics obtained rigorously in Section 2 for rotationally symmetric solutions should be stable for fully general solutions. (As noted above, "stable" is to be understood modulo the choices of time and location about which one renormalizes.) 


\subsection{The cylinder soliton}

Recall that a Ricci soliton is a tuple $\left(\mathcal{M}^{m}, g, X\right)$, where $\mathcal{M}^{m}$ is a smooth manifold, $g$ is its Riemannian metric and $X$ is a complete vector field on $\mathcal{M}^{m}$ such that the identity

$$
-2 \operatorname{Rc}(g)=\mathcal{L}_{X} g+\lambda g
$$

holds for some $\lambda \in\{-1,0,1\}$. It is well known that each Ricci soliton gives rise to a self-similar solution $\bar{g}(t)$ of Ricci flow

$$
\frac{\partial}{\partial t} \bar{g}=-2 \operatorname{Rc}(\bar{g})
$$

defined by

$$
\bar{g}(t)=(T+\lambda t)\left(\varphi_{t}^{*} g\right),
$$

where $\varphi_{t}$ is the 1-parameter family of diffeomorphisms generated by the vector fields $(T+\lambda t)^{-1} X$.

Now consider the manifold $\mathbb{R} \times S^{n}$ with local coordinates $\left(y^{0} ; y^{1}, \ldots, y^{n}\right)$, where $y^{0} \equiv x \in \mathbb{R}$ and $\left(y^{1}, \ldots, y^{n}\right) \equiv y \in S^{n}$. Let $\hat{g} \equiv g_{\text {can }}$ denote the round metric of unit radius on $S^{n}$. Define a product metric $g$ on $\mathbb{R} \times S^{n}$ by

$$
g=d x \otimes d x+2(n-1) \hat{g}
$$

noting that its Ricci curvature satisfies

$$
\operatorname{Rc}(g)=(n-1) \hat{g}
$$

Let $X$ be the vector field on $\mathbb{R} \times S^{n}$ defined by

$$
X=\operatorname{grad}\left(\frac{x^{2}}{4}\right)=\frac{x}{2} \frac{\partial}{\partial x},
$$

noting that the Lie derivative of $g$ with respect to $X$ is

$$
\mathcal{L}_{X} g=d x \otimes d x
$$

It is then easy to see that

$$
-2 \operatorname{Rc}(g)=\mathcal{L}_{X} g-g
$$

and hence that $\left(\mathbb{R} \times S^{n}, g, X\right)$ is a shrinking gradient Ricci soliton. We call this the cylinder soliton. 
For later use, we observe that the Levi-Civita connection of $g$ is given in local coordinates $\left(y^{0} ; y^{1}, \ldots, y^{n}\right)$ by the Christoffel symbols

$$
\Gamma_{i j}^{k}= \begin{cases}\hat{\Gamma}_{i j}^{k} & \text { if } 1 \leq i, j, k \leq n \\ 0 & \text { otherwise }\end{cases}
$$

Here and throughout the remainder of this paper, a hat designates a geometric quantity associated to the round unit sphere $\left(S^{n}, \hat{g}\right)$. The components $R_{i j k \ell}=g_{\ell m} R_{i j k}^{m}$ of the Riemannian curvature of $g$ are

$$
R_{i j k \ell}= \begin{cases}2(n-1) \hat{R}_{i j k \ell}=2(n-1)\left(\hat{g}_{i \ell} \hat{g}_{j k}-\hat{g}_{i k} \hat{g}_{j \ell}\right) & \text { if } 1 \leq i, j, k, \ell \leq n \\ 0 & \text { otherwise }\end{cases}
$$

and those of its Ricci tensor are

$$
R_{i j}= \begin{cases}\hat{R}_{i j}=(n-1) \hat{g}_{j k} & \text { if } 1 \leq i, j \leq n \\ 0 & \text { otherwise }\end{cases}
$$

\subsection{Dilated Ricci flow}

Motivated by the rigorous results obtained in Section 2, we want to study finite-time local singularities of Ricci flow that are modeled on the cylinder soliton (figure 2). Accordingly, let $\left(\mathcal{M}^{m}, G(t)\right)$ be a solution of Ricci flow

$$
\frac{\partial}{\partial t} G=-2 \operatorname{Rc}(G)
$$

that exists for $0 \leq t<T<\infty$. Given any vector field $X$ on $\mathcal{M}^{m}$, let $\varphi_{t}$ be the family of diffeomorphisms solving

$$
\frac{\partial}{\partial t} \varphi(\cdot, t)=\frac{1}{T-t} X(\varphi(\cdot, t)) .
$$

Then the blow-up of $G$ at $T$ modified by $X$ is the metric $g(t)$ defined by

$$
G(t)=(T-t)\left(\varphi_{t}^{*} g(t)\right)
$$

Observing that

$$
\frac{\partial}{\partial t}\left(\varphi_{t}^{*} g\right)=\frac{1}{T-t} \varphi_{t}^{*}\left(\mathcal{L}_{X} g\right)+\varphi_{t}^{*}\left(\frac{\partial}{\partial t} g\right)
$$




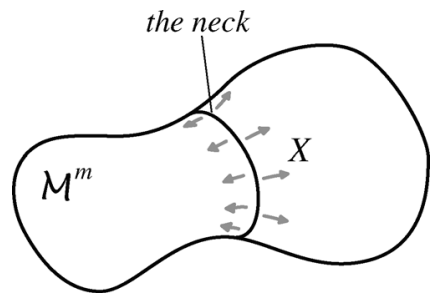

Figure 2: Approximating $X$ near an almost cylindrical neck.

one computes that

$$
\varphi_{t}^{*}(-2 \operatorname{Rc}(g))=-2 \operatorname{Rc}(G)=\frac{\partial}{\partial t} G=\varphi_{t}^{*}\left(-g+\mathcal{L}_{X} g+(T-t) \frac{\partial}{\partial t} g\right) .
$$

Thus if one regards $g$ as a function of the rescaled time variable

$$
\tau=\log \frac{1}{T-t}
$$

then $\left(\mathcal{M}^{m}, g(\tau), X\right)$ becomes a solution of the dilated Ricci flow:

$$
\frac{\partial}{\partial \tau} g=-2 \operatorname{Rc}(g)-\mathcal{L}_{X} g+g
$$

The following is an immediate consequence of Equations (3.4) and (3.10).

Lemma 3.1. The cylinder soliton $\left(\mathbb{R} \times S^{n}, g, X\right)$ is a stationary solution of the dilated Ricci flow.

\subsection{First and second order variation formulas}

If $h$ is a symmetric (2,0)-tensor on a Riemannian manifold $\left(\mathcal{M}^{m}, g\right)$, its Lichnerowicz Laplacian $\Delta_{\ell} h$ is defined by

$$
\Delta_{\ell} h_{i j}=\Delta h_{i j}+2 R_{i p q j} h^{p q}-R_{i}^{k} h_{k j}-R_{j}^{k} h_{i k},
$$

where $\Delta h$ is the rough Laplacian. Its divergence $\delta h$ is defined by

$$
(\delta h)_{j}=-\nabla^{i} h_{i j}
$$

The following variation formulas are proved by direct calculation. Note that first-order variation formulae are standard and are found, for instance, in [9] or [6]. 
Lemma 3.2. Let $\left(\mathcal{M}^{m}, g\right)$ be a Riemannian manifold; let $h$ be a symmetric $(2,0)$-tensor on $\mathcal{M}^{m}$; and let $\varepsilon$ be small enough that

$$
\tilde{g}=g+\varepsilon h
$$

is a Riemannian metric.

(1) In local coordinates, the Christoffel symbols $\tilde{\Gamma}$ of the Levi-Civita connection of $\tilde{g}$ are

$$
\begin{aligned}
\tilde{\Gamma}_{i j}^{k}= & \Gamma_{i j}^{k}+\frac{\varepsilon}{2}\left(\nabla_{i} h_{j}^{k}+\nabla_{j} h_{i}^{k}-\nabla^{k} h_{i j}\right) \\
& -\frac{\varepsilon^{2}}{2} h_{\ell}^{k}\left(\nabla_{i} h_{j}^{\ell}+\nabla_{j} h_{i}^{\ell}-\nabla^{\ell} h_{i j}\right)+\mathcal{O}\left(\varepsilon^{3}\right) .
\end{aligned}
$$

(2) In local coordinates, the Riemann tensor $\widetilde{\operatorname{Rm}}$ of $\tilde{g}$ is

$$
\begin{aligned}
\tilde{R}_{i j k}^{\ell}= & R_{i j k}^{\ell}+\frac{\varepsilon}{2}\left(\nabla_{i} \nabla_{k} h_{j}^{\ell}-\nabla_{i} \nabla^{\ell} h_{j k}-\nabla_{j} \nabla_{k} h_{i}^{\ell}+\nabla_{j} \nabla^{\ell} h_{i k}\right. \\
& \left.+R_{i j m}^{\ell} h_{k}^{m}-R_{i j k}^{m} h_{m}^{\ell}\right) \\
& +\frac{\varepsilon^{2}}{2} h_{m}^{\ell}\left(\nabla_{i} \nabla^{m} h_{j k}-\nabla_{j} \nabla^{m} h_{i k}-\nabla_{i} \nabla_{k} h_{j}^{m}+\nabla_{j} \nabla_{k} h_{i}^{m}\right) \\
& +\frac{\varepsilon^{2}}{4}\left(\nabla_{i} h_{k}^{m}+\nabla_{k} h_{i}^{m}-\nabla^{m} h_{i k}\right)\left(\nabla_{j} h_{m}^{\ell}+\nabla^{\ell} h_{j m}-\nabla_{m} h_{j}^{\ell}\right) \\
& -\frac{\varepsilon^{2}}{4}\left(\nabla_{j} h_{k}^{m}+\nabla_{k} h_{j}^{m}-\nabla^{m} h_{j k}\right)\left(\nabla_{i} h_{m}^{\ell}+\nabla^{\ell} h_{i m}-\nabla_{m} h_{i}^{\ell}\right) \\
& +\frac{\varepsilon^{2}}{2} h_{p}^{\ell}\left(R_{i j k}^{q} h_{q}^{p}-R_{i j q}^{p} h_{k}^{q}\right)+\mathcal{O}\left(\varepsilon^{3}\right) .
\end{aligned}
$$

(3) In local coordinates, the Ricci tensor $\widetilde{\mathrm{Rc}}$ of $\tilde{g}$ is

$$
\begin{aligned}
\tilde{R}_{i j}= & R_{i j}-\frac{\varepsilon}{2}\left[\Delta_{\ell} h_{i j}+\nabla_{i} \nabla_{j} H+\nabla_{i}(\delta h)_{j}+\nabla_{j}(\delta h)_{i}\right] \\
& +\frac{\varepsilon^{2}}{2} h^{p q}\left(\nabla_{p} \nabla_{q} h_{i j}+\nabla_{i} \nabla_{j} h_{p q}-\nabla_{i} \nabla_{p} h_{q j}-\nabla_{j} \nabla_{p} h_{q i}\right) \\
& +\frac{\varepsilon^{2}}{2}\left(\frac{1}{2} \nabla_{i} h_{p}^{q} \nabla_{j} h_{q}^{p}+\nabla_{p} h_{i}^{q} \nabla^{p} h_{q j}-\nabla_{p} h_{i}^{q} \nabla_{q} h_{j}^{p}\right) \\
& +\frac{\varepsilon^{2}}{2}\left(\frac{1}{2} \nabla_{k} H+(\delta h)_{k}\right)\left(\nabla_{i} h_{j}^{k}+\nabla_{j} h_{i}^{k}-\nabla^{k} h_{i j}\right) \\
& +\frac{\varepsilon^{2}}{2} h^{p q}\left(h_{i}^{k} R_{j p k q}+h_{j}^{k} R_{i p k q}+2 h_{q}^{k} R_{i p k j}\right)+\mathcal{O}\left(\varepsilon^{3}\right) .
\end{aligned}
$$


(4) If $X$ and $Y$ are vector fields on $\mathcal{M}^{m}$ and $\tilde{X}=X+\varepsilon Y$, then

$$
\mathcal{L}_{\tilde{X}} \tilde{g}=\mathcal{L}_{X} g+\varepsilon\left(\mathcal{L}_{Y} g+\mathcal{L}_{X} h\right)+\varepsilon^{2} \mathcal{L}_{Y} h
$$

in particular,

$$
\begin{aligned}
\left(\mathcal{L}_{\tilde{X}} \tilde{g}\right)_{i j}= & \nabla_{i} X_{j}+\nabla_{j} X_{i} \\
& +\varepsilon\left[\left(\nabla_{i} Y_{j}+\nabla_{j} Y_{i}\right)+\left(X^{k} \nabla_{k} h_{i j}+\nabla_{i} X^{k} h_{k j}+\nabla_{j} X^{k} h_{i k}\right)\right] \\
& +\varepsilon^{2}\left(Y^{k} \nabla_{k} h_{i j}+\nabla_{i} Y^{k} h_{k j}+\nabla_{j} Y^{k} h_{i k}\right) .
\end{aligned}
$$

\subsection{Arbitrary perturbations of a stationary solution}

Let $\left(\mathcal{M}^{m}, g, X\right)$ be any stationary solution of the dilated Ricci flow (3.10), and let $h$ be an arbitrary symmetric $(2,0)$-tensor small enough that the perturbation

$$
\tilde{g}=g+h
$$

is a Riemannian metric on $\mathcal{M}^{m}$. Denote the trace of $h$ with respect to $g$ by

$$
H=\operatorname{tr}_{g} h
$$

Let $Y$ be the vector field metrically dual to the 1 -form $\frac{1}{2} d H+\delta h$, namely

$$
Y=\left(\frac{1}{2} d H+\delta h\right)^{\sharp},
$$

and form the perturbation

$$
\tilde{X}=X+Y
$$

of the vector field $X$. The role of $Y$ is to implement a DeTurck trick $[11,12]$ that makes the linearization of the dilated Ricci flow (3.10) strictly parabolic. Now applying Lemma 3.2 gives the following result.

Lemma 3.3. The metric $\tilde{g}(\tau)$ will be a solution of dilated Ricci flow (3.10) for the vector field $\tilde{X}$ if and only if $h$ evolves by the nonlinear system

$$
\frac{\partial}{\partial \tau} h=\mathcal{A} h+\mathcal{Q}(h)+\mathcal{C}(h)
$$


where $\mathcal{A}$ is the linear elliptic operator defined by

$$
\mathcal{A} h=\Delta_{\ell} h-\mathcal{L}_{X} h+h,
$$

the quadratic term $\mathcal{Q}(h)$ is defined by

$$
[\mathcal{Q}(h)]_{i j}=h_{i}^{p} \nabla_{j} \nabla_{q} h_{p}^{q}+h_{j}^{p} \nabla_{i} \nabla_{q} h_{p}^{q}
$$

$$
\begin{aligned}
& +h^{p q}\left(\nabla_{i} \nabla_{p} h_{q j}+\nabla_{j} \nabla_{p} h_{q i}-\nabla_{p} \nabla_{q} h_{i j}-\nabla_{i} \nabla_{j} h_{p q}\right) \\
& -\frac{1}{2}\left(h_{i}^{k} \nabla_{j} \nabla_{k} H+h_{j}^{k} \nabla_{i} \nabla_{k} H+\nabla_{i} h_{j}^{k} \nabla_{k} H+\nabla_{j} h_{i}^{k} \nabla_{k} H\right)
\end{aligned}
$$$$
+\nabla_{i} h_{j}^{p} \nabla_{q} h_{p}^{q}+\nabla_{j} h_{i}^{p} \nabla_{q} h_{p}^{q}+\nabla_{p} h_{i}^{q} \nabla_{q} h_{j}^{p}
$$$$
-\nabla_{p} h_{i}^{q} \nabla^{p} h_{j q}-\frac{1}{2} \nabla_{i} h_{p}^{q} \nabla_{j} h_{q}^{p}
$$$$
+h^{p q}\left(h_{i}^{k} R_{j p q k}+h_{j}^{k} R_{i p q k}-2 h_{q}^{k} R_{i p k j}\right),
$$

and $\mathcal{C}(h)$ is at least third-order in $h$ and its covariant derivatives.

The cubic term $\mathcal{C}(h)$ will not be important in the formal asymptotic analysis that follows.

\subsection{The linearization at the cylinder soliton}

In the case that the stationary solution of the dilated Ricci flow is the cylinder soliton $\left(\mathbb{R} \times S^{n}, g, X\right)$ introduced in Section 3.1, the linear operator $\mathcal{A}$ defined in (3.18) can be written in a more useful form. To see this, we again work in the coordinate system $\left(y^{0} ; y^{1}, \ldots, y^{n}\right)$, with $y^{0} \equiv x \in \mathbb{R}$ and $\left(y^{1}, \ldots, y^{n}\right) \equiv y \in S^{n}$. Recalling formula (3.7), it is easy to see that

$$
h_{i j}-\left(R_{i}^{k} h_{k j}+R_{j}^{k} h_{i k}\right)= \begin{cases}h_{00} & \text { if } i=j=0, \\ \frac{h_{i 0}}{2} & \text { if } 1 \leq i \leq n, \\ \frac{h_{0 j}}{2} & \text { if } 1 \leq j \leq n, \\ 0 & \text { if } 1 \leq i, j \leq n .\end{cases}
$$


This observation allows one to write the Lie derivative of $h$ with respect to $X$ as

$$
\begin{aligned}
\left(\mathcal{L}_{X} h\right)_{i j} & =\frac{x}{2} \frac{\partial}{\partial x} h_{i j}+\frac{1}{2} h_{i j}\left(\delta_{i}^{0}+\delta_{j}^{0}\right) \\
& =\frac{x}{2} \frac{\partial}{\partial x} h_{i j}+h_{i j}-\left(R_{i}^{k} h_{k j}+R_{j}^{k} h_{i k}\right) .
\end{aligned}
$$

Then using the simple identity

$$
R_{i \ell} R_{j k}-R_{i k} R_{j \ell}=\frac{n-1}{2} R_{i j k \ell}
$$

one reaches the following conclusion.

Lemma 3.4. On the cylinder soliton, the linear operator $\mathcal{A}$ defined in (3.18) is given by

$$
(\mathcal{A} h)_{i j}=\Delta h_{i j}-\frac{x}{2} \frac{\partial}{\partial x} h_{i j}+\frac{4}{n-1}\left(R_{i j} R_{p q}-R_{i q} R_{p j}\right) h^{p q},
$$

where $\Delta h$ denotes the rough Laplacian.

\subsection{An upper bound for the spectrum of the linearization}

Since the manifold $\mathbb{R} \times S^{n}$ is noncompact, it is not necessarily the case that the formally elliptic operator $\mathcal{A}$ has discrete point spectrum. For our operator $\mathcal{A}$, the first-order term $-\frac{1}{2} x \partial_{x}$ makes this happen, as we will now show.

We consider the bundle $\mathcal{S}_{2}$ of $(2,0)$-tensors on $\mathbb{R} \times S^{n}$ and the space $C_{0}^{\infty}\left(\mathcal{S}_{2}\right)$ of smooth compactly supported sections of this bundle. The operator $\mathcal{A}$ maps this space to itself. We define the inner product

$$
(h, k) \doteqdot \int_{\mathbb{R}} \int_{S^{n}} h_{i j} k_{i j} e^{-x^{2} / 4} d \theta d x,
$$

where $d \theta$ is the volume form on $S^{n}$. We write $\|h\|=\sqrt{ }(h, h)$ for the corresponding norm. The completion of $C_{0}^{\infty}\left(\mathcal{S}_{2}\right)$ with respect to this norm is $L^{2}\left(\mathcal{S}_{2} ; e^{-x^{2} / 4} d \theta d x\right)$, which we abbreviate to $L^{2}$. (See [13], for instance.)

Lemma 3.5. The closure of the densely defined operator $\mathcal{A}$ is a self-adjoint operator with compact resolvent. $\mathcal{A}$ is bounded from above. Its spectrum consists of discrete finite multiplicity point spectrum. 
Proof. One can write $\mathcal{A}$ as

$$
(\mathcal{A} h)_{i j}=e^{x^{2} / 4} \frac{\partial}{\partial x}\left\{e^{-x^{2} / 4} \frac{\partial h_{i j}}{\partial x}\right\}+\frac{1}{2(n-1)} \hat{\Delta} h_{i j}+V_{i j p q} h^{p q}
$$

in which $\hat{\Delta}$ is the rough Laplacian in the direction tangential to the spheres $\{x\} \times S^{n}$ and

$$
V_{i j p q} \doteqdot \frac{4}{n-1}\left(R_{i j} R_{p q}-R_{i q} R_{j p}\right)
$$

By integration by parts, one finds that for any $h, k \in C_{0}^{\infty}\left(\mathcal{S}_{2}\right)$ one has

$$
(h,-\mathcal{A} k)=\int_{\mathbb{R}} \int_{S^{n}}\left\{\partial_{x} h_{i j} \partial_{x} k_{i j}+\frac{1}{2(n-1)} \hat{\nabla}_{\ell} h_{i j} \hat{\nabla}_{\ell} k_{i j}-V_{i j p q} h_{i j} k_{p q}\right\} e^{-x^{2} / 4} d \theta d x,
$$

from which it is evident that $\mathcal{A}$ is symmetric. Boundedness of $V_{i j p q}$ implies that there is a constant $C_{0}$ such that

$$
(h,-\mathcal{A} h) \geq \int_{\mathbb{R}} \int_{S^{n}}|\nabla h|^{2} e^{-x^{2} / 4} d \theta d x-C_{0}\|h\|^{2}
$$

holds for all $h \in C_{0}^{\infty}\left(\mathcal{S}_{2}\right)$.

Let $H^{1}=H^{1}\left(\mathcal{S}_{2}\right)$ be the completion of $C_{0}^{\infty}\left(\mathcal{S}_{2}\right)$ for the norm corresponding to the inner product

$$
\langle h, k\rangle \doteqdot \int_{\mathbb{R}} \int_{S^{n}}\left\{\nabla_{i} h_{p q} \nabla_{i} k_{p q}+h_{p q} k_{p q}\right\} e^{-x^{2} / 4} d \theta d x .
$$

(Again, see [13].) Then the standard Hilbert space arguments show that for all $\lambda>C_{0}$, the equation $\lambda h-\mathcal{A} h=k$ has a distributional solution $h \in H^{1}$ for any $k \in L^{2}$. It follows that the closure of $\mathcal{A}$ is a self-adjoint operator in $L^{2}$ whose domain

$$
\mathrm{D}(\mathcal{A})=\left\{(\lambda-\mathcal{A})^{-1} k \mid k \in L^{2}\right\}
$$

is contained in $H^{1}$. (Here it makes no difference which $\lambda>C_{0}$ one chooses.) In fact, the inequality (3.23) implies that the $H^{1}$ norm is equivalent with the norm $\||| h \mid\|^{2}=\langle h,(\lambda-\mathcal{A}) h\rangle$, so that $H^{1}$ is the form domain of $\mathcal{A}$, i.e., $H^{1}=$ $\mathrm{D}(\sqrt{\lambda-\mathcal{A}})$

The inequality (3.23) tells us that the spectrum of $\mathcal{A}$ is contained in the interval $\left(-\infty, C_{0}\right]$. 
To see that $\mathcal{A}$ has compact resolvent, we recall Lemma 2.7, which implies

$$
\|x h\|^{2} \leq C \int_{\mathbb{R}} \int_{S^{n}}\left\{\left|\partial_{x} h\right|^{2}+|h|^{2}\right\} e^{-x^{2} / 4} d \theta d x \leq C\|h\|_{H^{1}}^{2}
$$

Hence

$$
\|h\|^{2}+\|x h\|^{2}+\|\nabla h\|^{2} \leq C(h,(\lambda-\mathcal{A}) h)
$$

This inequality together with the Rellich-Kondrachov theorem imply that the imbedding $H^{1} \subset L^{2}$ is compact. Since $H^{1}$ is the form domain of $\mathcal{A}$, we conclude that $\mathcal{A}$ indeed has compact resolvent, so that its spectrum is pure point spectrum.

Remark. More generally, the linearization of the modified Ricci flow operator at any gradient Ricci soliton $\left(\mathcal{M}^{n}, g\right)$ will be self-adjoint in $L^{2}\left(\mathcal{M}^{n}, g ; e^{-\varphi} d \mu\right)$ if $d \mu$ is the measure naturally associated to $g$ and one takes $\varphi$ to be the soliton potential function.

\subsection{A decomposition into invariant subspaces}

In order to compute the spectrum of $\mathcal{A}$, we split the space $L^{2}\left(\mathcal{S}_{2}\right)$ into a number of $\mathcal{A}$-invariant subspaces.

It is a standard fact that any $(2,0)$-tensor $h$ on a Riemannian manifold $\left(\mathcal{M}^{m}, g\right)$ admits the decomposition

$$
h=h^{\vee}+h^{\circ}
$$

where

$$
h^{\vee}=\left(\operatorname{tr}_{g} h\right) g
$$

is a multiple of the metric, and

$$
h^{\circ}=h-\frac{1}{m} h^{\vee}
$$

is trace free.

One can exploit the product structure of the cylinder soliton $\left(\mathbb{R} \times S^{n}, g, X\right)$ to obtain a more refined decomposition. Again using coordinates $\left(y^{0} ; y^{1}, \ldots, y^{n}\right)$ and adopting the convention that Roman indices lie in the range $0 \ldots n$ while Greek indices lie in $1 \ldots n$, we decompose a given 
symmetric $(2,0)$-tensor $h$ on $\mathbb{R} \times S^{n}$ as

$h=M d x \otimes d x+N_{\alpha}\left(d x \otimes d y^{\alpha}+d y^{\alpha} \otimes d x\right)+\left(O_{\alpha \beta}^{\vee}+O_{\alpha \beta}^{\circ}\right) d y^{\alpha} \otimes d y^{\beta}$

$$
=M d x \otimes d x+N_{\alpha}\left(d x \otimes d y^{\alpha}+d y^{\alpha} \otimes d x\right)+\left(P \hat{g}_{\alpha \beta}+Q_{\alpha \beta}\right) d y^{\alpha} \otimes d y^{\beta} .
$$

Here one should regard $M$ as a scalar function on $\mathbb{R} \times S^{n}, N(x, \cdot)$ as a 1-parameter family of 1 -forms defined on the spheres $\{x\} \times S^{n}, P$ as the scalar function

$$
P=\frac{2(n-1)}{n}(H-M)
$$

and $Q(x, \cdot)$ as the 1-parameter family of trace-free $(2,0)$-tensors defined on the spheres $\{x\} \times S^{n}$ by

$$
Q_{\alpha \beta}=h_{\alpha \beta}-P \hat{g}_{\alpha \beta}
$$

The utility of this decomposition is that it exhibits a set of invariant subspaces for $\mathcal{A}$. To demonstrate this, it will be helpful to fix additional notation. Let $\hat{\Delta}$ denote the rough Laplacian of the canonical sphere metric $\hat{g}$. Let $-\hat{\Delta}_{d R}$ denote its Hodge-de Rham Laplacian, recalling that $\hat{\Delta}_{d R}$ acts on a 1 -form $N$ by

$$
\hat{\Delta}_{d R} N_{\alpha}=-[(d \delta+\delta d) N]_{\alpha}=\hat{\Delta} N_{\alpha}-\hat{R}_{\alpha}^{\beta} N_{\beta}=\hat{\Delta} N_{\alpha}-(n-1) N_{\alpha}
$$

Let $\hat{\Delta}_{\ell}$ denote the Lichnerowicz Laplacian of $\hat{g}$, which acts on a trace-free tensor $Q$ by

$$
\hat{\Delta}_{\ell} Q_{\alpha \beta}=\hat{\Delta} Q_{\alpha \beta}-2 n Q_{\alpha \beta}
$$

Finally, let $\mathcal{B}$ denote the differential operator defined by

$$
\mathcal{B}=\frac{\partial^{2}}{\partial x^{2}}-\frac{x}{2} \frac{\partial}{\partial x}=e^{x^{2} / 4} \frac{\partial}{\partial x} e^{-x^{2} / 4} \frac{\partial}{\partial x}
$$


Using these conventions, one computes using Equation (3.20) that the decomposition of $\mathcal{A} h$ corresponding to $(3.24(\mathrm{a}) /(\mathrm{b}))$ is

$$
\begin{aligned}
(\mathcal{A} h)_{00} & =\left[\mathcal{B}+\frac{1}{2(n-1)} \hat{\Delta}\right] M \\
(\mathcal{A} h)_{\alpha 0} & =\left[\mathcal{B}+\frac{1}{2(n-1)} \hat{\Delta}\right] N_{\alpha}=\left[\mathcal{B}+\frac{1}{2(n-1)} \hat{\Delta}_{d R}+\frac{1}{2}\right] N_{\alpha} \\
(\mathcal{A} h)_{\alpha \beta}^{\vee} & =\left\{\left[\mathcal{B}+\frac{1}{2(n-1)} \hat{\Delta}+1\right] P\right\} \hat{g}_{\alpha \beta} \\
(\mathcal{A} h)_{\alpha \beta}^{\circ} & =\left[\mathcal{B}+\frac{1}{2(n-1)} \hat{\Delta}-\frac{1}{n-1}\right] Q_{\alpha \beta} .
\end{aligned}
$$

This calculation proves the following result.

Lemma 3.6. On the cylinder soliton, the operator $\mathcal{A}$ given by (3.20) may be represented schematically with respect to the decomposition (3.24) as

$$
\mathcal{A}:\left(\begin{array}{c}
M \\
N \\
P \\
Q
\end{array}\right) \longmapsto\left(\begin{array}{c}
{\left[\mathcal{B}+\frac{1}{2(n-1)} \hat{\Delta}\right] M} \\
{\left[\mathcal{B}+\frac{1}{2(n-1)} \hat{\Delta}_{d R}+\frac{1}{2}\right]} \\
{\left[\mathcal{B}+\frac{1}{2(n-1)} \hat{\Delta}+1\right] P} \\
{\left[\mathcal{B}+\frac{1}{2(n-1)} \hat{\Delta}-\frac{1}{n-1}\right] Q}
\end{array}\right) .
$$

The only contribution to the spectrum of $\mathcal{A}$ comes from its four component operators: $\mathcal{B}+1 /(2(n-1)) \hat{\Delta}$ and $\mathcal{B}+1 /(2(n-1)) \hat{\Delta}+1$ acting on scalar functions, $\mathcal{B}+1 /(2(n-1)) \hat{\Delta}_{d R}+1 / 2$ acting on 1 -forms and $\mathcal{B}+1 /(2(n-1)) \hat{\Delta}-1 /(n-1)$ acting on trace-free $(2,0)$-tensors.

\subsection{The spectrum of the linearization}

The decomposition obtained in Lemma 3.6 allows us to analyze the spectrum of $\mathcal{A}$ using separation of variables. To illustrate the idea, suppose that

$$
M(x, y)=h(x) F(y)
$$

where $h$ is an eigenfunction of $\mathcal{B}$ with eigenvalue $\lambda$, and $F$ is an eigenfunction of $1 / 2(n-1) \hat{\Delta}$ with eigenvalue $\mu$. Then

$$
\left[\mathcal{B}+\frac{1}{2(n-1)} \hat{\Delta}\right] M=(\lambda+\mu) M
$$


This line of argument leads easily to the following observation.

Lemma 3.7. All eigenvalues of $\mathcal{A}: \mathrm{D}(\mathcal{A}) \rightarrow L^{2}$ have the form $\lambda+\mu$, where $\lambda$ is an eigenvalue of $\mathcal{B}$ acting on $\mathfrak{h}=L^{2}\left(\mathbb{R} ; e^{-x^{2} / 4}\right)$, and $\mu$ is an eigenvalue of either $1 / 2(n-1) \hat{\Delta}$ or $1 / 2(n-1) \hat{\Delta}+1$ acting on $C^{\infty}\left(S^{n}\right)$, of $1 / 2(n-1) \hat{\Delta}_{d R}+1 / 2$ acting on $\Omega_{1}\left(S^{n}\right)$, or of $1 / 2(n-1) \hat{\Delta}-1 / n-1$ acting on the space of smooth trace-free $(2,0)$-tensors on $S^{n}$.

To make this result useful, we need some simple facts about the component operators that appear in $(3.26)$.

The operator $\mathcal{B}=\partial^{2} / \partial x^{2}-(x / 2)(\partial / \partial x)$ acting on scalar functions. The Hermite polynomials $\left\{h_{k}\right\}_{k=0}^{\infty}$ constitute a complete orthogonal family for the operator $\mathcal{B}$ on the weighted Hilbert space

$$
\mathfrak{h}=L^{2}\left(\mathbb{R}, e^{-x^{2} / 4} d x\right) .
$$

Moreover, one has

$$
(\mathcal{B}+1) h_{k}=\left(1-\frac{k}{2}\right) h_{k},
$$

so that the spectrum of $\mathcal{B}$ is $\left\{0,-\frac{1}{2},-1,-\frac{3}{2}, \ldots\right\}$. (Notice that $\mathfrak{h}$ is a larger space than we defined in Section 2, since we are not restricting to even functions here.)

The operator $1 / 2(n-1) \hat{\Delta}$ acting on scalar functions. It is well known that the spectrum of $\hat{\Delta}$ acting on $C^{\infty}\left(S^{n}\right)$ is $\left\{\lambda_{k}\right\}_{k \geq 0}$, where

$$
\lambda_{k}=-k(n+k-1)
$$

In particular, the only non-negative eigenvalue of $1 / 2(n-1) \hat{\Delta}$ is $\lambda_{0}=0$. Its eigenspace consists of the constant functions.

The operator $1 / 2(n-1) \hat{\Delta}+1$ acting on scalar functions. If $k \geq 2$, then

$$
\lambda_{k}+2(n-1)=(2-k)(n-1)-k^{2} \leq-k^{2}<0 .
$$

Hence the only possible non-negative eigenvalues of $1 /(2(n-1)) \hat{\Delta}+1$ are

$$
\frac{\lambda_{0}+2(n-1)}{2(n-1)}=1
$$

and

$$
\frac{\lambda_{1}+2(n-1)}{2(n-1)}=\frac{1}{2} \frac{n-2}{n-1} \in\left[0, \frac{1}{2}\right) .
$$


The eigenspace corresponding to 1 consists of constants. The eigenspace corresponding to $1 / 2(n-2) /(n-1)$ consists of the spherical harmonics: the restrictions to $S^{n}$ of the linear functions on $\mathbb{R}^{n+1}$.

The operator $1 / 2(n-1) \hat{\Delta}_{d R}+1 / 2$ acting on 1 -forms. It is shown in [8] that the spectrum of $\hat{\Delta}_{d R}$ acting on the space $\Omega_{1}\left(S^{n}\right)$ of smooth 1 -forms is

$$
\left\{\lambda_{k}\right\}_{k \geq 1} \bigcup\left\{\lambda_{k}+2-n\right\}_{k \geq 1}
$$

If $k \geq 1$, then $\lambda_{k}+n-1 \leq-1$. Because $\lambda_{k}+2-n \leq \lambda_{k}$ in all dimensions $n \geq 2$, it follows that $\frac{1}{2(n-1)} \hat{\Delta}_{d R}+\frac{1}{2}$ is negative definite.

The operator $1 / 2(n-1) \hat{\Delta}-1 /(n-1)$ acting on trace-free $(2,0)$-tensors. The operator $1 /(2(n-1)) \hat{\Delta}-1 /(n-1)$ is clearly negative definite.

Combining these observations with Lemma 3.7 leads to the following conclusion.

Lemma 3.8. On the cylinder soliton, the only possible non-negative eigenvalues of the linearization $\mathcal{A}$ are $0,1 / 2(n-2) /(n-1), 1 / 2$, and 1 .

- The eigenspace corresponding to 1 consists of constant multiples of $\hat{g}$.

- The eigenspace corresponding to $1 / 2$ consists of multiples of $\hat{g}$ that are linear in $x \in \mathbb{R}$.

- The eigenspace corresponding to $1 / 2(n-2) /(n-1)$ consists of spherical harmonics.

- The eigenspace corresponding to 0 consists of constant functions and of multiples of $\hat{g}$ that are quadratic in $x \in \mathbb{R}$. (When $n=2$, the spherical harmonics also belong to this eigenspace.)

Remark. The results above can be obtained in another way. Since all geometric data of the metric (3.2) are independent of $x \in \mathbb{R}$, one has the simple commutator

$$
\left[\mathcal{A}, \frac{\partial}{\partial x}\right]=\frac{1}{2} \frac{\partial}{\partial x} .
$$

So if $\mathcal{A} h=\lambda h$, a straightforward induction argument shows that

$$
\mathcal{A}\left(\frac{\partial^{k}}{\partial x^{k}} h\right)=\left(\lambda+\frac{k}{2}\right)\left(\frac{\partial^{k}}{\partial x^{k}} h\right)
$$

for all integers $k \geq 0$. Since Lemma 3.5 proves that the spectrum of $\mathcal{A}$ is bounded from above, it follows readily that for every eigentensor $h$ of 
$\mathcal{A}$, there exists some integer $\kappa=\kappa(h) \geq 0$ and a family of (2,0)-tensors $\hat{h}_{1}, \ldots, \hat{h}_{\kappa}$ independent of $x \in \mathbb{R}$ such that

$$
h=\sum_{k=0}^{\kappa} x^{k} \hat{h}_{k} .
$$

\subsection{Our Ansatz}

The analysis in Section 3.8 shows that any eigenvalues of $\mathcal{A}$ corresponding to trace-free (i.e., nonrotationally symmetric) perturbations of the cylinder soliton are all strictly negative. As we explained in the introduction to this section, the only perturbations relevant to our formal center-manifold computation are those corresponding to null eigenvalues of the linearization. Therefore, we adopt the Ansatz that

$$
h=M d x \otimes d x+N_{\alpha}\left(d x \otimes d y^{\alpha}+d y^{\alpha} \otimes d x\right)+\left(P \hat{g}_{\alpha \beta}+Q_{\alpha \beta}\right) d y^{\alpha} \otimes d y^{\beta}
$$

has the special form

$$
h(x, y, \tau)=u(x, \tau) d x \otimes d x+\{v(x, \tau)+w(y, \tau)\} \hat{g}
$$

where once again, $\tau$ denotes rescaled time, $x$ denotes a point in $\mathbb{R}$, and $y$ denotes a point in $S^{n}$. Specifically - continuing to let $\left\{h_{k}\right\}_{k=0}^{\infty}$ denote the Hermite polynomials — we assume that $M \equiv u$, where

$$
u(x, \tau)=u_{0}(\tau) h_{0}(x)=u_{0}(\tau)
$$

We further assume that $N \equiv 0$, that $Q \equiv 0$, and that $P \equiv v+w$, where

$$
v(x, \tau)=v_{2}(\tau) h_{2}(x)=v_{2}(\tau)\left(x^{2}-2\right)
$$

and

$$
w(y, \tau)= \begin{cases}\sum_{i=1}^{3} \omega_{i}(\tau) \Omega_{i}(y) & \text { if } n=2 \\ 0 & \text { otherwise. }\end{cases}
$$

Here $\left(\Omega_{1}, \Omega_{2}, \Omega_{3}\right)$ is a basis for the space of spherical harmonics on $S^{2}$. (Recall that $w$ corresponds to a positive eigenvalue of the linearization whenever the total dimension $n+1$ is at least 4.) 
To implement our formal center-manifold analysis, we shall study the flow

$$
\frac{\partial}{\partial \tau} h=\mathcal{A} h+\mathcal{Q}(h)
$$

that models Equation (3.17) up to second order. To do so requires us to analyze the quadratic term $\mathcal{Q}(h)$ at the cylinder soliton in a manner analogous to what was done above for the linearization $\mathcal{A}$. To simplify the notation, we again use coordinates $\left(y^{0} ; y^{1}, \ldots, y^{n}\right)$ and assume that Roman indices lie in the range $0 \ldots n$ while Greek indices lie in $1 \ldots n$. If $f$ is a smooth function, we further adopt the convention that $f_{x}=\partial f / \partial x$ and $f_{\alpha}=\partial f / \partial y^{\alpha}$.

We shall begin with computations that are more general than what we need at the moment. To wit, we assume only that $h$ has the form given by (3.27), without imposing the specific assumptions (3.28)-(3.30). One then verifies readily that all first-covariant derivatives of the tensor $h$ vanish except

$$
\begin{aligned}
\nabla_{0} h_{00} & =u_{x} \\
\nabla_{0} h_{\alpha \beta} & =v_{x} \hat{g}_{\alpha \beta} \\
\nabla_{\gamma} h_{\alpha \beta} & =w_{\gamma} \hat{g}_{\alpha \beta} .
\end{aligned}
$$

All second-covariant derivatives of $h$ vanish except

$$
\begin{aligned}
\nabla_{0} \nabla_{0} h_{00} & =u_{x x} \\
\nabla_{0} \nabla_{0} h_{\alpha \beta} & =v_{x x} \hat{g}_{\alpha \beta} \\
\nabla_{\gamma} \nabla_{\delta} h_{\alpha \beta} & =\hat{\nabla}_{\gamma} \hat{\nabla}_{\delta} w \hat{g}_{\alpha \beta} .
\end{aligned}
$$

Moreover, the only nonvanishing derivatives of the trace

$$
H=g^{i j} h_{i j}=u+\frac{n}{2(n-1)}(v+w)
$$

are

$$
\begin{aligned}
& \nabla_{0} H=u_{x}+\frac{n}{2(n-1)} v_{x} \\
& \nabla_{\gamma} H=\frac{n}{2(n-1)} w_{\gamma}
\end{aligned}
$$


and

$$
\begin{aligned}
& \nabla_{0} \nabla_{0} H=u_{x x}+\frac{n}{2(n-1)} v_{x x}, \\
& \nabla_{\gamma} \nabla_{\delta} H=\frac{n}{2(n-1)} \hat{\nabla}_{\gamma} \hat{\nabla}_{\delta} w .
\end{aligned}
$$

Using these formulas, a straightforward calculation reveals that the first component of the quadratic term

$$
\begin{aligned}
{[\mathcal{Q}(h)]_{00}=} & 2 h_{00} \nabla_{0} \nabla_{0} h_{00}-h^{\alpha \beta} \nabla_{0} \nabla_{0} h_{\alpha \beta}-h_{00} \nabla_{0} \nabla_{0} H \\
& -\nabla_{0} h_{00} \nabla_{0} H+\frac{3}{2}\left(\nabla_{0} h_{00}\right)^{2}-\frac{1}{2} \nabla_{0} h_{\alpha}^{\beta} \nabla_{0} h_{\beta}^{\alpha}
\end{aligned}
$$

may be written as

$$
\begin{aligned}
{[\mathcal{Q}(h)]_{00}=} & u u_{x x}-\frac{n}{4(n-1)^{2}} v_{x x}[2(n-1) u+v+w] \\
& +\frac{1}{2} u_{x}^{2}-\frac{n}{8(n-1)^{2}} v_{x}\left[4(n-1) u_{x}+v_{x}\right]
\end{aligned}
$$

Then using the fact that

$$
g^{\gamma \delta} \hat{\nabla}_{\gamma} \hat{\nabla}_{\delta} w=\frac{1}{2(n-1)} \hat{\Delta} w=-\frac{n}{2(n-1)} w
$$

one computes that the remaining components

$$
\begin{aligned}
{[\mathcal{Q}(h)]_{\alpha \beta}=} & h_{\alpha}^{\gamma} \nabla_{\beta} \nabla_{\delta} h_{\gamma}^{\delta}+h_{\beta}^{\gamma} \nabla_{\alpha} \nabla_{\delta} h_{\gamma}^{\delta}-h^{00} \nabla_{0} \nabla_{0} h_{\alpha \beta} \\
& +h^{\gamma \delta}\left(\nabla_{\alpha} \nabla_{\gamma} h_{\delta \beta}+\nabla_{\beta} \nabla_{\gamma} h_{\delta \alpha}-\nabla_{\gamma} \nabla_{\delta} h_{\alpha \beta}-\nabla_{\alpha} \nabla_{\beta} h_{\gamma \delta}\right) \\
& -\frac{1}{2}\left(h_{\alpha}^{\gamma} \nabla_{\beta} \nabla_{\gamma} H+h_{\beta}^{\gamma} \nabla_{\alpha} \nabla_{\gamma} H+\nabla_{\alpha} h_{\beta}^{\gamma} \nabla_{\gamma} H+\nabla_{\beta} h_{\alpha}^{\gamma} \nabla_{\gamma} H\right) \\
& +\nabla_{\alpha} h_{\beta}^{\gamma} \nabla_{\delta} h_{\gamma}^{\delta}+\nabla_{\beta} h_{\alpha}^{\gamma} \nabla_{\delta} h_{\gamma}^{\delta}+\nabla_{\gamma} h_{\alpha}^{\delta} \nabla_{\delta} h_{\beta}^{\gamma} \\
& -\nabla_{0} h_{\alpha}^{\gamma} \nabla^{0} h_{\beta \gamma}-\nabla_{\delta} h_{\alpha}^{\gamma} \nabla^{\delta} h_{\beta \gamma}-\frac{1}{2} \nabla_{\alpha} h_{\gamma}^{\delta} \nabla_{\beta} h_{\delta}^{\gamma} \\
& +h^{\gamma \delta}\left(h_{\alpha}^{\eta} R_{\beta \gamma \delta \eta}+h_{\beta}^{\eta} R_{\alpha \gamma \delta \eta}-2 h_{\delta}^{\eta} R_{\alpha \gamma \eta \beta}\right)
\end{aligned}
$$


are given by

$$
\begin{aligned}
{[\mathcal{Q}(h)]_{\alpha \beta}=} & -\frac{1}{2(n-1)}\left\{2(n-1) u v_{x x}+v_{x}^{2}\right\} \hat{g}_{\alpha \beta} \\
& -\frac{1}{4(n-1)^{2}}\left\{|\hat{\nabla} w|_{\hat{g}}^{2}-n w(v+w)\right\} \hat{g}_{\alpha \beta} \\
& -\frac{n-2}{8(n-1)^{2}}\left\{4(v+w) \hat{\nabla}_{\alpha} \hat{\nabla}_{\beta} w+3 w_{\alpha} w_{\beta}\right\} .
\end{aligned}
$$

\subsection{The inner layer $|x|=\mathrm{o}(\sqrt{\tau})$}

We now derive a formal profile of a solution

$$
\tilde{g}(x, y, \tau)=g(x, y)+h(x, y, \tau)
$$

near a singularity modeled on the cylinder soliton $g$. We assume that (3.27) and (3.28)-(3.30) hold. These assumptions force $h$ to belong to the kernel of the linearization of equation (3.17), because

$$
\mathcal{A} h=[\mathcal{B} u] d x \otimes d x+[(\mathcal{B}+1) v] \hat{g}=0 .
$$

Recall that $w$ vanishes by assumption if $n>2$. In this context, (3.32) becomes

$$
[\mathcal{Q}(h)]_{00}=-\left\{v_{2} w+\frac{n}{(n-1)^{2}}\left[(n-1) u_{0} v_{2}+v_{2}^{2}\right]\right\} h_{0}-\left\{\frac{n}{(n-1)^{2}} v_{2}^{2}\right\} h_{2} .
$$

And (3.33) reduces to

$$
[\mathcal{Q}(h)]_{\alpha \beta}=\mathcal{P} \hat{g}_{\alpha \beta},
$$

where

$$
\mathcal{P}=\left\{\frac{1}{2} w^{2}-\frac{1}{4}|\hat{\nabla} w|_{\hat{g}}^{2}-2 u_{0} v_{2}-\frac{4}{n-1} v_{2}^{2}\right\} h_{0}+\left\{\frac{1}{2} v_{2} w-\frac{2}{n-1} v_{2}^{2}\right\} h_{2} .
$$

In order to derive ODE efficiently for the functions $u_{0}, v_{2}$, and $\omega_{1}, \omega_{2}, \omega_{3}$ from Equations (3.34) and (3.35), it is helpful to adopt some additional 
notation: if $f$ is a function of $y \in S^{n}$, we define

$$
\|f\|_{S^{n}}^{2}=\int_{S^{n}} f(y)^{2} d \hat{\mu}
$$

and if $X$ and $Y$ are symmetric (2,0)-tensors on $\mathbb{R} \times S^{n}$, we define

$$
\langle\langle X, Y\rangle\rangle=\int_{-\infty}^{\infty}\langle X, Y\rangle_{x} e^{-x^{2} / 4} d x
$$

where $\langle X, Y\rangle_{x}=\int_{\{x\} \times S^{n}}\langle X, Y\rangle_{\hat{g}} d \hat{\mu}$. By using the fact that $\left\{h_{k}\right\}_{k=0}^{\infty}$ is an orthogonal family for $\mathfrak{h}$, it is then easy to see that

$$
\begin{aligned}
u_{0}^{\prime}(\tau) \cdot\left\|h_{0}\right\|_{\mathfrak{h}}^{2}\|1\|_{S^{n}}^{2} & =\frac{d}{d t}\left\langle\left\langle h, h_{0} d x^{2}\right\rangle\right\rangle \\
& =\left\langle\left\langle\mathcal{Q}(h), h_{0} d x^{2}\right\rangle\right\rangle \\
& =-\frac{n}{(n-1)^{2}}\left[(n-1) u_{0} v_{2}+v_{2}^{2}\right] \cdot\left\|h_{0}\right\|_{\mathfrak{h}}^{2}\|1\|_{S^{n}}^{2}
\end{aligned}
$$

and

$$
\begin{aligned}
v_{2}^{\prime}(\tau) \cdot n\left\|h_{2}\right\|_{\mathfrak{h}}^{2}\|1\|_{S^{n}}^{2} & =\frac{d}{d t}\left\langle\left\langle h, h_{2} \hat{g}\right\rangle\right\rangle \\
& =\left\langle\left\langle\mathcal{Q}(h), h_{2} \hat{g}\right\rangle\right\rangle \\
& =-\frac{2}{n-1} v_{2}^{2} \cdot n\left\|h_{2}\right\|_{\mathfrak{h}}^{2}\|1\|_{S^{n}}^{2}
\end{aligned}
$$

Similarly, for any $i \in\{1,2,3\}$, we find when $n=2$ that

$$
\omega_{i}^{\prime}(\tau) \cdot 2\left\|h_{0}\right\|_{\mathfrak{h}}^{2}\left\|\Omega_{i}\right\|_{S^{2}}^{2}=\frac{d}{d t}\left\langle\left\langle h, \Omega_{i} \hat{g}\right\rangle\right\rangle=\left\langle\left\langle\mathcal{Q}(h), \Omega_{i} \hat{g}\right\rangle\right\rangle=0 .
$$

This calculations let us derive a formal profile of $h$ in the inner layer $|x|=\mathrm{o}(\sqrt{\tau})$.

Lemma 3.9. For perturbations of the form

$$
h=\left\{u_{0}(\tau) h_{0}(x)\right\} d x \otimes d x+\left\{v_{2}(\tau) h_{2}(x)+\sum_{i=1}^{3} \omega_{i}(\tau) \Omega_{i}(y)\right\} \hat{g}
$$


the flow (3.31) is equivalent to the system of $\mathrm{ODE}$

$$
\begin{aligned}
\frac{d}{d \tau} u_{0} & =-\frac{n}{(n-1)^{2}}\left[(n-1) u_{0} v_{2}+v_{2}^{2}\right] \\
\frac{d}{d \tau} v_{2} & =-\frac{2}{n-1} v_{2}^{2} \\
\frac{d}{d t} \omega_{i} & =0 \quad(i=1,2,3)
\end{aligned}
$$

whose solutions, up to an error term of $\mathrm{o}\left(\tau^{-1}\right)$ as $\tau \rightarrow \infty$, are

$$
\begin{aligned}
& u_{0}(\tau)=\left\{\begin{array}{l}
-\frac{\log (2 \tau)}{2 \tau}+\frac{c}{\tau} \quad \text { if } n=2, \\
-\frac{n}{2(n-2)} \frac{1}{\tau} \quad \text { otherwise }
\end{array}\right. \\
& v_{2}(\tau)=\frac{n-1}{2 \tau} \\
& \omega_{i}(\tau)=\omega_{i}(0) \quad(i=1,2,3) .
\end{aligned}
$$

Formally, this is the profile of $h$ in the region $|x|=\mathrm{o}(\sqrt{\tau})$.

Proof. If $v_{2}$ is not identically zero, Equation (3.37) has the explicit solution

$$
v_{2}(\tau)=\frac{n-1}{c+2 \tau}
$$

Then Equation (3.36) becomes

$$
\frac{d}{d \tau} u_{0}(\tau)+\left(\frac{n}{c+2 \tau}\right) u_{0}=-\frac{n}{(c+2 \tau)^{2}} .
$$

An integrating factor for this linear ODE is $(c+2 \tau)^{n / 2}$, whence one obtains

$$
u_{0}(\tau)= \begin{cases}-\frac{\log (c+2 \tau)}{c+2 \tau}+\frac{C}{c+2 \tau} & \text { if } n=2, \\ -\frac{n}{n-2} \frac{1}{c+2 \tau}+\frac{C}{(c+2 \tau)^{n / 2}} & \text { otherwise. }\end{cases}
$$

Remark. To compare the formal results above with those obtained rigorously in Section 2, observe that our choices of dilating factors imply that 
the quantity $a_{2}(\tau)$ appearing in Section 2 and the quantity $v_{2}(\tau)$ appearing above are related by

$$
a_{2}(\tau) \approx \frac{v_{2}(\tau)}{4(n-1)} \approx \frac{1}{8 \tau},
$$

because in the inner layer, we have $x \approx \sigma$ and

$$
\begin{aligned}
\left\{2(n-1)+v_{2}(\tau)\right. & \left.h_{2}(x)+\mathrm{o}\left(\tau^{-1}\right)\right\} \hat{g} \\
= & 2(n-1)\left[1+a_{2}(\tau) h_{2}(\sigma)+\mathrm{o}\left(\tau^{-1}\right)\right]^{2} \hat{g} \\
& =\left\{2(n-1)+4(n-1) a_{2}(\tau) h_{2}(\sigma)+\mathrm{o}\left(\tau^{-1}\right)\right\} \hat{g} .
\end{aligned}
$$

Remark. By modifying $\tilde{g}$ by an initial conformal diffeomorphism, one may make all $\omega_{i}$ vanish. Therefore, throughout the remainder of this paper, we will assume that $\omega_{i} \equiv 0$ for $i=1,2,3$.

\subsection{The intermediate layer $|x|=\mathcal{O}(\sqrt{\tau})$}

In order to study the region where $|x|=\mathcal{O}(\sqrt{\tau})$, we replace the coordinates $(x, \tau)$ with $(\xi, \tau)$, where

$$
\xi=\frac{x}{\sqrt{\tau}}
$$

If $f$ is a smooth function of $(x, \tau)$, we define $F(\xi, \tau)=f(x, \tau)$. Then one has the formulas

$$
f_{\tau}=F_{\tau}-\frac{\xi}{2 \tau} F_{\xi} \quad \text { and } \quad f_{x}=\frac{1}{\sqrt{\tau}} F_{\xi}
$$

Let

$$
U(\xi, \tau)=u(x, \tau) \quad \text { and } \quad V(\xi, \tau)=v(x, \tau),
$$

where $u$ and $v$ are the quantities that appear in the simple Ansatz (3.27). Then substituting formulas (3.32) and (3.33) into the PDE (3.31), one computes that

$$
U_{\tau}=-\frac{\xi}{2} U_{\xi}+\frac{1}{\tau}\left\{\begin{array}{c}
(1+U) U_{\xi \xi}+\frac{\xi}{2} U_{\xi}+\frac{1}{2} U_{\xi}^{2} \\
-\frac{n}{4(n-1)^{2}} V_{\xi \xi}[2(n-1) U+V] \\
-\frac{n}{8(n-1)^{2}} V_{\xi}\left[4(n-1) U_{\xi}+V_{\xi}\right]
\end{array}\right\}
$$


and

$$
V_{\tau}=V-\frac{\xi}{2} V_{\xi}+\frac{1}{\tau}\left\{V_{\xi \xi}(1-U)+\frac{\xi}{2} V_{\xi}-\frac{1}{2(n-1)} V_{\xi}^{2}\right\} .
$$

Recalling Lemma 3.9, we will construct an approximate solution by considering the equations

$$
U_{\tau}=-\frac{\xi}{2} U_{\xi}+\mathrm{o}\left(\tau^{-1}\right)
$$

and

$$
V_{\tau}=V-\frac{\xi}{2} V_{\xi}+\mathcal{O}\left(\tau^{-2}\right)
$$

These equations suggest that the solution is modeled by the time-independent profiles $U(\xi) \equiv C_{1}$ and $V(\xi)=C_{2} \xi^{2}$, respectively. Matching this layer with the results of Lemma 3.9 determines $C_{1}$ and $C_{2}$, yielding the following result.

Lemma 3.10. In the intermediate layer $|x / \sqrt{\tau}|=|\xi|=\mathcal{O}(1)$, the formal solution is modeled by

$$
u(x, \tau) \approx U\left(\frac{x}{\sqrt{\tau}}\right) \equiv 0 \quad \text { and } \quad v(x, \tau) \approx V\left(\frac{x}{\sqrt{\tau}}\right)=\frac{n-1}{2} \frac{x^{2}}{\tau}
$$

To wit,

$$
\tilde{g} \approx d x \otimes d x+2(n-1)\left[1+(\xi / 2)^{2}\right] \hat{g}
$$

\subsection{The outer layer $|x|=\mathrm{o}(1 / \sqrt{T-t})$}

To describe the outer layer, we first introduce the "blown-down" coordinate

$$
s=e^{-\tau / 2} x=\sqrt{T-t} \cdot x,
$$

which should be compared to the metric distance $s$ defined in Section 2 .

If one were to follow the method above by defining $\bar{U}(s, \tau)=u(x, \tau)$ and $\bar{V}(s, \tau)=v(x, \tau)$, an easy computation would show that

$$
\bar{U}_{\tau}=\mathcal{O}(T-t) \quad \text { and } \quad \bar{V}_{\tau}=V+\mathcal{O}(T-t)
$$

Unfortunately, the steady-state solutions to these equations fail to convey enough useful information.

Instead, we proceed as follows. Fix a large number $\Xi>0$. For each $s$, let $t_{s}$ be the time such that $|\xi|=\Xi$ at $t_{s}$, where $\xi$ is defined by (3.42). Let 
$\tau_{s}=\log 1 /\left(1-t_{s}\right)$. Then

$$
\Xi=|\xi|=\frac{|x|}{\sqrt{\tau_{s}}}=\frac{|s|}{\sqrt{\left(T-t_{s}\right) \tau_{s}}} .
$$

This shows that

$$
\tau_{s}=\log \left(\Xi^{2}\right)+\log \frac{1}{s^{2}}+\log \tau_{s}
$$

hence that

$$
\tau_{s}=[2+\mathrm{o}(1)] \log \frac{1}{|s|} \quad \text { as }|s| \rightarrow 0 .
$$

Now we "blow-down" the solution of the dilated ("blown-up") Ricci by defining

$$
\underline{u}(s, \tau)=e^{-\tau} u(x, \tau) \text { and } \underline{v}(s, \tau)=e^{-\tau} v(x, \tau) .
$$

Then at time $t_{s}$, we compute that

$$
\underline{v}\left(s, \tau_{s}\right)=e^{-\tau_{s}} V\left(\frac{x}{\sqrt{\tau_{s}}}\right)=\frac{n-1}{2} \frac{s^{2}}{\tau_{s}} .
$$

When $|s|$ is small and $\Xi$ is large, the undilated solution $\underline{v}$ cannot change much in the short time $t_{s} \leq t<T$. Hence (3.44) implies that as $|s| \rightarrow 0$, one has

$$
\begin{aligned}
\underline{v}\left(s, \tau_{s}\right) & =\frac{n-1}{2} \frac{s^{2}}{[2+\mathrm{o}(1)] \log 1 /|s|}[1+\mathrm{o}(1)] \\
& =\frac{n-1}{2} \frac{s^{2}}{\log 1 / s^{2}}[1+\mathrm{o}(1)] .
\end{aligned}
$$

Since $\underline{u}(s, \tau)=e^{-\tau} U(x / \sqrt{\tau}) \equiv 0$, we have obtained the following result.

Lemma 3.11. In the outer layer $|x|=\mathrm{o}\left(\frac{1}{\sqrt{T-t}}\right)$, the formal solution is modeled by

$$
u(x, \tau) \approx \frac{1}{T-t} \underline{u}(s, \tau) \quad \text { and } \quad v(x, \tau) \approx \frac{1}{T-t} \underline{v}(s, \tau),
$$

where

$$
\underline{u}(s, \tau) \equiv 0 \quad \text { and } \quad \underline{v}(s, \tau)=\frac{n-1}{2} \frac{s^{2}}{\log 1 / s^{2}}[1+\mathrm{o}(1)] .
$$


To wit,

$$
v(x, \tau) \approx \frac{n-1}{2} \frac{x^{2}}{\tau+\log 1 / x^{2}} .
$$

\section{References}

[1] S. Altschuler, S.B. Angenent and Y. Giga, Mean curvature flow through singularities for surfaces of rotation, J. Geom. Anal. 5 (3) (1995), 293-358.

[2] S.B. Angenent and D.G. Aronson, Non-axial self-similar hole filling for the porous medium equation, J. Amer. Math. Soc. 14 (4) (2001), 737782.

[3] S.B. Angenent and D. Knopf, An example of neckpinching for Ricci flow on $S^{n+1}$, Math. Res. Lett. 11 (4) (2004), 493-518.

[4] S.B. Angenent and J.J.L. Velázquez, Asymptotic shape of cusp singularities in curve shortening, Duke Math. J. 77 (1) (1995), 71-110.

[5] S.B. Angenent and J.J.L. Velázquez, Degenerate neckpinches in mean curvature flow, J. Reine Angew. Math. 482 (1997), 15-66.

[6] A.L. Besse, Einstein manifolds, Ergebnisse der Mathematik und ihrer Grenzgebiete (3), 10, Springer-Verlag, Berlin, 1987.

[7] O. Biquard, Métriques d'Einstein asymptotiquement symétriques, Astérisque 265, Société Mathématique de France, 2000.

[8] M. Boucetta, Spectre des laplaciens de Lichnerowicz sur les sphères et les projectifs réels, [Spectra of Lichnerowicz Laplacians on spheres and real projective spaces] Publ. Mat. 43 (2) (1999), 451-483.

[9] B. Chow and D. Knopf, The Ricci flow: An introduction, Mathematical Surveys and Monographs, 110, American Mathematical Society, Providence, RI, 2004.

[10] P. Daskalopoulos and N. Šešum, Type II extinction profile of maximal solutions to the Ricci flow in $\mathbb{R}^{2}$, Preprint, arXiv:math.AP/0606288.

[11] D.M. DeTurck, Deforming metrics in the direction of their Ricci tensors, J. Differential Geom., 18 (1) (1983), 157-162.

[12] D.M. DeTurck, Deforming metrics in the direction of their Ricci tensors (improved version) in Collected papers on Ricci flow, 
ed. H.-D. Cao, B. Chow, S.-C. Chu and S.-T. Yau, Internat. Press, Somerville, MA, 2003.

[13] J. Eichhorn and J. Fricke, The module structure theorem for Sobolev spaces on open manifolds, Math. Nachr. 194 (1998), 35-47.

[14] M. Feldman, T. Ilmanen and D. Knopf, Rotationally symmetric shrinking and expanding gradient Kähler-Ricci solitons, J. Differential Geom. 65 (2) (2003), 169-209.

[15] S. Filippas and R.V. Kohn, Refined asymptotics for the blowup of $u_{t}-\Delta u=u^{p}$, Comm. Pure Appl. Math. 45 (7) (1992), 821-869.

[16] A. Friedman and B. McLeod, Blow-up of positive solutions of semilinear heat equations, Indiana Univ. Math. J. 34 (2) (1985), 425-447.

[17] Y. Giga and R.V. Kohn, Asymptotically self-similar blow-up of semilinear heat equations, Comm. Pure Appl. Math. 38 (3) (1985), 297-319.

[18] Y. Giga and R.V. Kohn, Characterizing blowup using similarity variables, Indiana Univ. Math. J. 36 (1) (1987), 1-40.

[19] Y. Giga and R.V. Kohn, Nondegeneracy of blowup for semilinear heat equations, Comm. Pure Appl. Math. 42 (6) (1989), 845-884.

[20] V.A. Galaktionov and S.A. Posashkov, Application of new comparison theorems to the investigation of unbounded solutions of nonlinear parabolic equations (Russian) Differentsialc nye Uravneniya 22 (7) (1986), 1165-1173, 1285.

[21] R.S. Hamilton, Three-manifolds with positive Ricci curvature, J. Differential Geom. 17 (2) (1982), 255-306.

[22] M.A. Herrero and J.J.L. Velázquez, Blow-up behaviour of onedimensional semilinear parabolic equations, Ann. Inst. H. Poincaré Anal. Non Linéaire 10 (2) (1993), 131-189.

[23] M.A. Herrero and J.J.L. Velázquez, Explosion de solutions d'équations paraboliques semilinéaires supercritiques [Blowup of solutions of supercritical semilinear parabolic equations] C. R. Acad. Sci. Paris Sér. I Math. 319 (2) (1994), 141-145.

[24] G. Huisken and C. Sinestrari, Convexity estimates for mean curvature flow and singularities of mean convex surfaces, Acta Math. 183 (1) (1999), 45-70. 
[25] T. Ilmanen and D. Knopf, A lower bound for the diameter of solutions to the Ricci flow with nonzero $H^{1}\left(M^{n} ; \mathbb{R}\right)$, Math. Res. Lett. 10 (2) (2003), 161-168.

[26] J.R. King, Self-similar behaviour for the equation of fast nonlinear diffusion, Philos. Trans. Roy. Soc. London Ser. A 343 (1993), 337-375.

[27] C.E. Mueller and F.B. Weissler, Single point blow-up for a general semilinear heat equation, Indiana Univ. Math. J. 34 (4) (1985), 881-913.

[28] G. Perelman, The entropy formula for the Ricci flow and its geometric applications, Preprint, arXiv:math.DG/0211159.

[29] N. Šešum, Curvature tensor under the Ricci flow, Amer. J. Math. 127 (6) (2005), 1315-1324.

[30] M. Simon, A class of Riemannian manifolds that pinch when evolved by Ricci flow, Manuscripta Math. 101 (1) (2000), 89-114.

[31] M. Simon, Deformation $C^{0}$ Riemannian metrics in the direction of their Ricci curvature, Comm. Anal. Geom. 10 (5) (2002), 1033-1074.

[32] P. Topping, Diameter control under Ricci flow, Comm. Anal. Geom. 13 (5) (2005), 1039-1055.

[33] J.B. van den Berg, J. Hulshof and J.R. King, Formal asymptotics of bubbling in the harmonic map heat flow, SIAM J. Appl. Math. 63 (5) (2003), 1682-1717 (electronic).

[34] F.B. Weissler, Single point blow-up for a semilinear initial value problem, J. Differential Equations 55 (2) (1984), 204-224.

UNIVERSITY OF WISCONSIN

MADISON

WISCONSIN

USA

E-mail address: angenent@math.wisc.edu

$U R L:$ http://www.math.wisc.edu/ ${ }^{\sim}$ angenent/

University of TeXas at Austin

Austin

TEXAS

USA

E-mail address: danknopf@math.utexas.edu

$U R L$ : http://www.ma.utexas.edu/ danknopf

ReCeived May 25, 2006 